ARTICLE

\title{
Listeria exploits IFITM3 to suppress antibacterial activity in phagocytes
}

Joel M. J. Tan (10 1,2, Monica E. Garner ${ }^{1,2}$, James M. Regeimbal ${ }^{3}$, Catherine J. Greene ${ }^{4}$, Jorge D. Rojas Márquez ${ }^{1}$, Dustin A. Ammendolia (1) 1,5, Adam R. R. McCluggage ${ }^{1}$, Taoyingnan Li ${ }^{1,5}$, Katherine J. Wu ${ }^{3}$, Marija Cemma ${ }^{1,5}$, Philip P. Ostrowski ${ }^{1,6}$, Brian Raught ${ }^{7,8}$, Michael S. Diamond (10) 9, Sergio Grinstein (D) 1,2,6, Robin M. Yates (iD) ${ }^{4}$, Darren E. Higgins (iD ${ }^{3,11 凶}$ \& John H. Brumell (1) 1,2,5,10,11凶

The type I interferon (IFN) signaling pathway has important functions in resistance to viral infection, with the downstream induction of interferon stimulated genes (ISG) protecting the host from virus entry, replication and spread. Listeria monocytogenes $(\mathrm{Lm})$, a facultative intracellular foodborne pathogen, can exploit the type I IFN response as part of their pathogenic strategy, but the molecular mechanisms involved remain unclear. Here we show that type I IFN suppresses the antibacterial activity of phagocytes to promote systemic $\mathrm{Lm}$ infection. Mechanistically, type I IFN suppresses phagosome maturation and proteolysis of $\mathrm{Lm}$ virulence factors ActA and LLO, thereby promoting phagosome escape and cell-to-cell spread; the antiviral protein, IFN-induced transmembrane protein 3 (IFITM3), is required for this type I IFN-mediated alteration. Ifitm $3^{-/-}$mice are resistant to systemic infection by $L m$, displaying decreased bacterial spread in tissues, and increased immune cell recruitment and pro-inflammatory cytokine signaling. Together, our findings show how an antiviral mechanism in phagocytes can be exploited by bacterial pathogens, and implicate IFITM3 as a potential antimicrobial therapeutic target.

\footnotetext{
${ }^{1}$ Cell Biology Program, Hospital for Sick Children, Toronto, ON, Canada. ${ }^{2}$ Institute of Medical Science, University of Toronto, Toronto, ON, Canada. ${ }^{3}$ Department of Microbiology, Blavatnik Institute, Harvard Medical School, Boston, MA, USA. ${ }^{4}$ Department of Biochemistry and Molecular Biology, University of Calgary, Calgary, AB, Canada. ${ }^{5}$ Department of Molecular Genetics, University of Toronto, Toronto, ON, Canada. ${ }^{6}$ Department of Biochemistry, University of Toronto, Toronto, ON, Canada. ${ }^{7}$ Princess Margaret Cancer Centre, University Health Network, Toronto, ON, Canada. ${ }^{8}$ Department of Medical Biophysics, University of Toronto, Toronto, ON, Canada. ${ }^{9}$ Departments of Medicine, Molecular Microbiology, Pathology \& Immunology, Washington University School of Medicine, St. Louis, MO, USA. ${ }^{10}$ SickKids IBD Centre, Hospital for Sick Children, Toronto, ON, Canada. ${ }^{11}$ These authors contributed equally: Darren E. Higgins, John H. Brumell. 凶email: dhiggins@hms.harvard.edu; john.brumell@sickkids.ca
} 
isteria monocytogenes $(\mathrm{Lm})$ is a facultative intracellular pathogen that can cause systemic infection in mammalian hosts. After ingestion by immune cells such as macrophages, $L m$ secretes virulence factors that can lyse the bacteriacontaining phagosome and escape into the cytosol ${ }^{1,2}$. Once in the cytosol, $\mathrm{Lm}$ is able to interact with host actin-related protein (Arp) $2 / 3$ complex using its virulence factor actin assemblyinducing protein (ActA) to polymerize actin and form actin comet tails to propel itself throughout the cell ${ }^{3}$. Motility is critical for $L m$ pathogenesis as it allows the bacteria to avoid host cell autophagic machinery and the ubiquitin-proteasome system ${ }^{4-6}$. To spread from cell-to-cell, motile $\mathrm{Lm}$ initiate formation of cellsurface filopodia (called protrusions) that can be engulfed by neighboring cells and mediate bacterial transfer without exposure to the extracellular space ${ }^{7}$.

Type 1 interferons (IFNs) are a subgroup of interferon proteins that help regulate innate and adaptive immune responses during viral and bacterial infections ${ }^{8-10}$. $L m$ infection is able to induce a robust type I IFN response during infection. Rupture of the bacteria-containing phagosome leads to the cytosolic delivery of cyclic dinucleotides, DNA and other factors that induce type I IFN production ${ }^{11-13}$. $\mathrm{Lm}$ is known to benefit from the type I IFN response, as evidenced by the finding that mice deficient in type I IFN receptors and regulatory factors are protected from $\mathrm{Lm}$ infection ${ }^{14-16}$. However, the molecular mechanisms behind bacterial exploitation of type I IFN signaling remain unclear.

Type I IFN is known to alter many aspects of the immune response to bacteria ${ }^{17}$. For example, $L m$-induced type 1 IFN production negatively regulates chemokine signaling and modulates neutrophil and monocyte recruitment ${ }^{18,19}$. We previously showed that type I IFN also promotes $\mathrm{Lm}$ cell-to-cell spread ${ }^{20}$. The early stages of actin-based motility, a process involving Arp2/ 3-mediated actin polymerization on the bacterial surface, were enhanced by type I IFN by mechanisms that remain unclear. As the type I IFN transcriptional response involves hundreds of genes $^{21}$, it is also not clear which ISGs promote $L m$ infection. This led us to examine how type I IFN affects the early stages of bacterial infection in macrophages, an important cellular target of these bacteria during systemic disease.

In this study, we examine the impact of type I IFN on phagosomal maturation and bacterial killing. We identify IFITM3 as a downstream target of type I IFN activation that suppresses proteolytic activity in phagocytes, thereby promoting $\mathrm{Lm}$ cell-tocell spread in macrophages. Cells and mice lacking IFITM3 display an increase in ActA degradation which leads to a decrease in $\mathrm{Lm}$ infection. Our study has important implications for understanding the role of type I IFN and IFITM3 in innate immunity during bacterial infections.

\section{Results}

Type I IFN impairs macrophage killing of phagosome-confined bacteria. To determine if type I IFN affects the antimicrobial activity of macrophages, we examined the fate of two different bacteria confined to phagosomes. We used the gram-positive $\mathrm{Lm}$ lacking the pore-forming toxin listeriolysin O (LLO, encoded by $h l y$ ). Since listeriolysin $\mathrm{O}$ is required for phagosome escape in macrophages, this mutant strain is confined to phagosomes and does not undergo intracellular growth ${ }^{22}$. As a model of gramnegative bacteria, we also examined Escherichia coli DH10B, a non-pathogenic lab strain. Bone marrow-derived macrophages (BMDMs) from wild-type (WT) C57BL/6 mice were treated with interferon beta (IFN $\beta$ ) for 20 hours (h) prior to exposure to bacteria. The uptake of bacteria was unaffected by IFN $\beta$ treatment (Supplementary Fig. 1a, b). The number of viable intracellular bacteria was quantified over a $24 \mathrm{~h}$ time course, and we observed a reduced bacterial load over time for both bacterial strains in untreated (UT) BMDMs, consistent with antibacterial activity in macrophage phagosomes (Fig. 1a, b). However, the intracellular bacterial load did not decrease in cells treated with IFN $\beta$ during this time course, though this effect was lost in IFN $\beta$-treated BMDMs from mice lacking the type I IFN receptor (Ifnar $1^{-1-}$, hereafter IFNAR1 KO). These findings suggest the type I IFN response suppresses antibacterial activity in phagosomes.

Next, we examined the impact of type I IFN on phagosome maturation. IFN $\beta$-treated cells showed reduced localization of the lysosome transmembrane protein LAMP1 to phagosomes compared to control cells (Fig. 1c-f). This effect was readily seen at late times (after $4 \mathrm{~h}$ ) post phagocytosis, indicating that sustained LAMP1 delivery was affected by type I IFN. However, this effect was not apparent at the earliest time points examined $(1 \mathrm{~h})$, indicating that early phagosome maturation events were not affected. Consistent with this finding, rapid delivery of internalized bacteria and dextrancontaining endosomes to acidified late endocytic compartments, visualized by pre-loading cells with cresyl violet, was not affected by IFN $\beta$ treatment (Supplementary Fig. $2 \mathrm{a}-\mathrm{d}$ ). Using a real-time fluorometric FRET-based assay to measure phagosome maturation, we also found that IFN $\beta$ treatment had no effect on phagosome fusion with endocytic compartments over a $2 \mathrm{~h}$ time course (Supplementary Fig. 2e). These results suggest that type I IFN affects delivery of some lysosomal components to bacteriacontaining phagosomes but does not affect early phagosome interactions with existing endocytic compartments.

By using fluorescence ratiometric imaging of CFSE-conjugated particles to measure the calibrated $\mathrm{pH}$ value of phagosomes, we determined that the rate of acidification in IFN $\beta$-treated BMDMs was unaffected (Supplementary Fig. 1c). Similarly, we found that the steady-state $\mathrm{pH}$ of lysosomes measured using FITC-dextran was not affected by IFN $\beta$ treatment (Supplementary Fig. 1d). Therefore, these data indicate that type I IFN does not affect the acidification of phagosomes or lysosomes.

Type I IFN suppresses phagosome proteolysis. Phagosomal proteases contribute to killing and digestion of internalized microbes $^{23}$. It is known that type II IFN (IFN $\gamma$ ) treatment can suppress phagosomal proteolysis to promote antigen presentation ${ }^{24-26}$. This phenotype is mediated by the NOX2 NADPH oxidase, a multisubunit complex that generates reactive oxygen species (ROS) within the lumen of the phagosome. ROS-mediated oxidation of critical cysteine residues in phagosome-localized proteases, such as cathepsin B and S, contributes to suppression of phagosomal proteolysis ${ }^{27}$, along with other potential mechanisms ${ }^{28}$. Since IFN $\gamma$ is known to promote bacterial killing 29,30 , ROS-mediated suppression of phagosomal proteolysis may be compensated by the direct antimicrobial activity of $\operatorname{ROS}^{31}$.

We examined phagosomal proteolytic activity in type I IFNtreated BMDMs. We utilized a real-time fluorometric assay using a dequenchable probe conjugated directly to silica beads ${ }^{32}$. IFN $\beta$ treated cells showed a deficiency in phagosomal proteolysis, comparable to the effect seen with IFN $\gamma$ treatment (Fig. 1g, h). This is consistent with a decrease in bacteria associated with DQBSA, a marker of proteolytically active compartments, in IFN $\beta$ treated BMDMs (Supplementary Fig. 2f, g). However, cells treated with IFN $\beta$ displayed decreased ROS production following phagocytosis relative to IFN $\gamma$ treatment and to untreated control cells (Fig. 1i). These findings indicate that type I IFN can suppress phagosomal proteolysis by a mechanism independent of ROS generation.

To further determine the effects of type I IFN on phagosome proteolysis, we examined the phagosome proteome using mass 

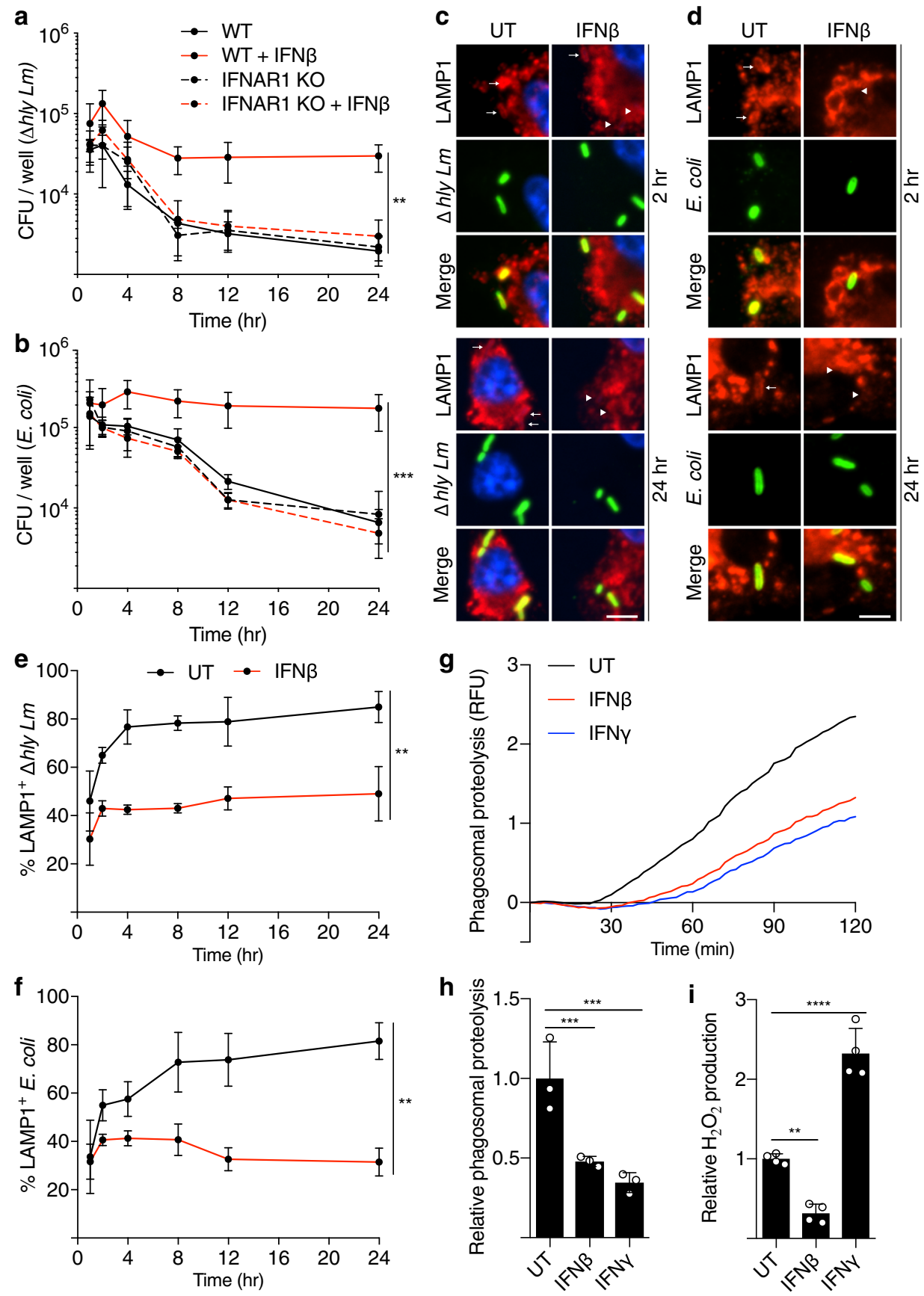

Fig. 1 Type I IFN suppresses antibacterial activity in phagosomes. Time course of bacteria killing over $24 \mathrm{~h}$ of (a) $\Delta h l y L m$ or (b) E. coli infected WT and IFNAR1 KO BMDMs with or without IFN $\beta$ pretreatment. ${ }^{\star \star} P=0.0012,{ }^{\star \star \star} P=0.001$. Representative images (c, d) and quantification (e, f) of LAMP1positive bacteria in BMDMs treated with or without IFN $\beta$, infected with $\Delta$ hly Lm (c, e) or E. coli (d, f) over $24 \mathrm{~h}$. ${ }^{\star \star} P<0.0087$. Arrow denotes LAMP1positive bacteria, arrowhead denotes LAMP1-negative bacteria. Bulk proteolysis over time (g) and slope of the curve's linear portion (h) of DQ-BSA-labeled particles in untreated (UT) phagosomes, or treated with IFN $\beta$ or IFN $\gamma$. ${ }^{\star \star \star} P<0.0009$. RFU relative fluorescence unit. $\mathbf{i}$ Measurement of ROS production in phagosomes of zymosan-fed BMDMs with and without IFN $\beta$ or IFN $\gamma$. ${ }^{\star \star} P=0.0042,{ }^{\star \star \star \star} P<0.0001$. Data shown are means \pm standard deviation (s.d.) for at least $n=3$ independent experiments. $P$ value was calculated using $(\mathbf{a}, \mathbf{b})$ two-way analysis of variance (ANOVA) and $(\mathbf{e}, \mathbf{f}, \mathbf{h}, \mathbf{i})$ one-way ANOVA. Scale bars, $3 \mu \mathrm{m}$.

spectrometry. Phagosomes were isolated from IFN $\beta$-treated BMDMs using magnetic latex beads. In separate experiments, we isolated lysosomes using iron dextran. We observed that proteolytic enzymes, as well as fusion and trafficking factors, were decreased in phagosomes from IFN $\beta$-treated BMDMs (Fig. 2a). In contrast, lysosomal protein contents were largely unaffected by IFN $\beta$ treatment. This data suggests that type I IFN inhibits the delivery of proteolytic enzymes to the phagosome during the maturation process.

Type I IFN inhibits phagosomal proteolysis of $\mathrm{Lm}$ virulence factors. Phagosomal proteases can degrade bacterial virulence factors, limiting their ability to alter host cell machinery ${ }^{33}$. Therefore, we hypothesized that type I IFN-mediated suppression 

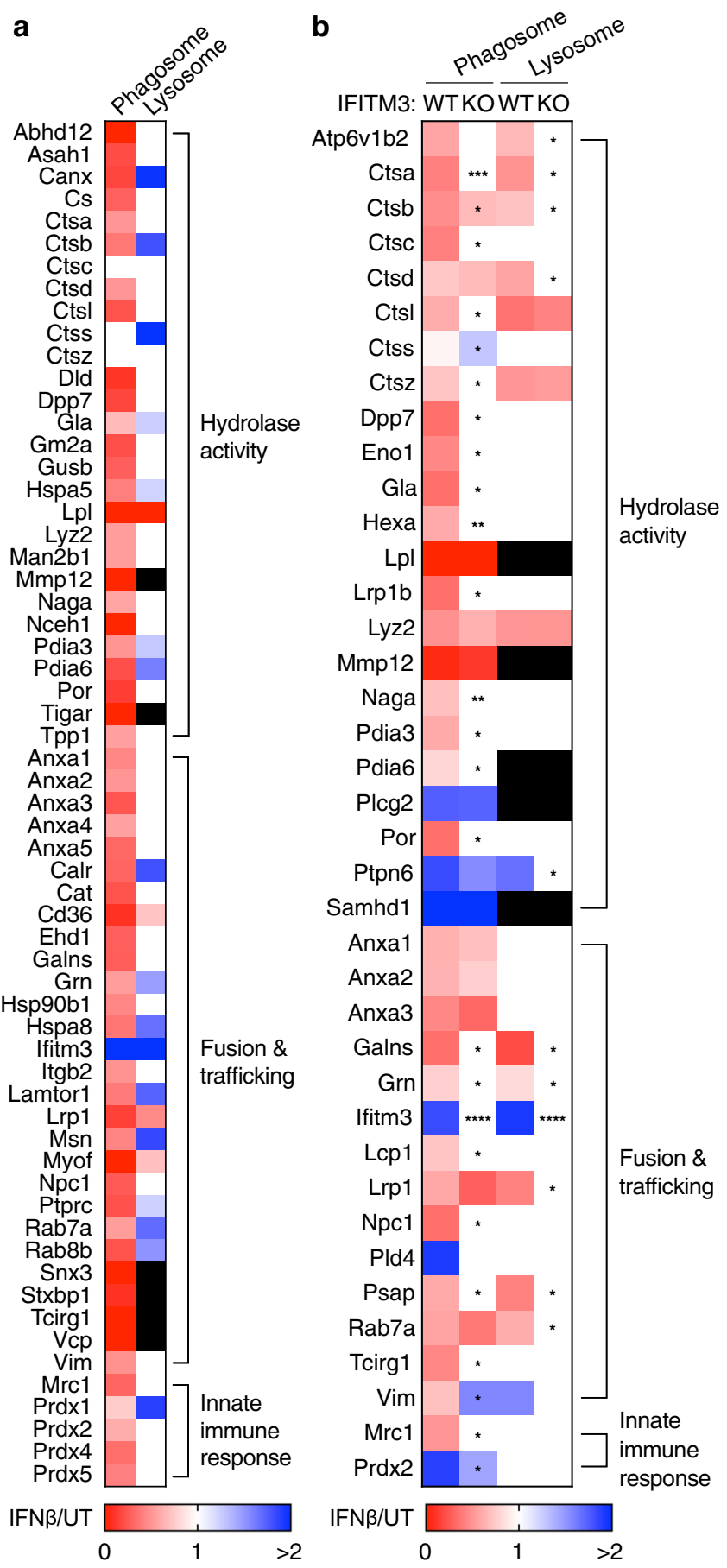

Fig. 2 Type I IFN inhibits the delivery of hydrolase activity-related proteins to phagosomes. Proteomics data obtained by mass spectrometry of phagosomes and lysosomes isolated from (a) WT BMDMs and (b) IFITM3 KO BMDMs. Legend denotes relative protein abundance between IFN $\beta$ treatment and UT controls. Black box indicates undetected proteins. Data shown are means for at least $n=3$ independent experiments. $P$ value was calculated using two-way ANOVA. ${ }^{\star} P<0.05,{ }^{\star \star} P<0.01$, ${ }^{\star \star \star} P<0.001$, ${ }_{\star \star \star \star \star} P<0.0001$

of phagosomal proteolysis would lead to enhanced activity of bacterial virulence factors within this compartment. During WT $\mathrm{Lm}$ infection, a number of virulence factors, including LLO and two phospholipases $\mathrm{C}$, act cooperatively to modify phagosome maturation and promote bacterial escape into the cytosol ${ }^{1}$. ActA also acts cooperatively with these virulence factors in the phagosome. ActA is a bacterial cell surface-associated virulence factor that was originally identified for its role in actin-based motility of $\mathrm{Lm}$ in the cytosol of host cells - a requirement for direct cell-to-cell spread by these bacteria ${ }^{34}$. Further studies revealed a role for ActA in promoting phagosome escape ${ }^{35}$.

To visualize ActA expression and cell surface localization during infection we generated fluorescent reporter strains (Fig. 3a, Supplementary Fig. 3a). Transcription of act $A$ was detected by green fluorescence protein (GFP) expression under the act $A$ promoter, in a cassette stably integrated in the tRNA ${ }^{A R G}$ locus of the bacterial chromosome. This cassette contains all of the known enhancer elements for ActA expression ${ }^{36}$ and we used the enhanced mutant of GFP for optimal detection of actA transcription (Supplementary Fig. 3a). The endogenous copy of actA within Listeria Pathogenicity Island 1 (LIPI-1) was left intact. Bacteria surface-localized ActA was detected by immunofluorescence using affinity-purified antibodies. The actA:GFP reporter strain was generated in the WT $L m$ background as well as the $\Delta h l y$ mutant and a mutant lacking virulence factors required for phagosomal escape $(\Delta h l y, \Delta p l c A$ and $\Delta p l c B)$, hereafter referred to as the triple knockout (TKO) $L m$ strain (Fig. 3a). ActA expression was previously thought to be initiated only after bacterial escape from the phagosome ${ }^{37,38}$. However, we observed that $\mathrm{Lm}$ expression of ActA can occur within phagosomes of J774 macrophages, prior to escape into the cytosol (Supplementary Fig. 3b).

In control BMDMs and RAW264.7 cells infected with the WT actA:GFP reporter strain, the majority of bacteria were localized to the cytosol at $4 \mathrm{~h}$ post-infection (p.i.), and displayed robust transcription of actA, visualized by the expression of actA:GFP and localization of ActA protein on the bacterial cell surface, as expected (Fig. 3b, Supplementary Fig. 3d, e). In contrast, $\Delta$ hly and TKO Lm trapped in LAMP1-positive phagolysosomes lacked surface ActA, despite high levels of GFP fluorescence, indicative of active actA:GFP expression (Supplementary Fig. 3d, f). We considered the possibility that ActA is degraded by proteases in phagosomes under these conditions. In support of this hypothesis, treatment of cells with broad-spectrum protease inhibitors promoted surface expression of ActA in phagosome-trapped bacteria (Fig. 3c). Furthermore, inhibition of phagosomal acidification and proteolysis with the vacuolar-type proton ATPase inhibitor bafilomycin $\mathrm{Al}^{39}$, had a similar effect (Fig. 3b, c, Supplementary Fig. 3c). Our findings indicate that ActA is actively degraded by phagosomal proteases prior to bacterial escape into the cytosol under normal infection conditions.

Next, we examined the impact of type I IFN treatment on ActA expression during $L m$ infection. BMDMs were treated with IFN $\beta$ prior to infection with actA:GFP reporter strains (for immunofluorescence imaging) and WT and TKO $\mathrm{Lm}$ strains (for protein analysis). Under these conditions, ActA localized to the bacterial surface in phagosome-trapped bacteria ( $\Delta h l y$ and TKO Lm) (Fig. 3b, c), suggesting that type I IFN-mediated suppression of phagosomal proteolysis stabilizes ActA. To further examine virulence factor expression during infection, we utilized a cell fractionation approach that separates bacterial cell surface proteins from secreted proteins (Fig. 4a). Western blotting revealed higher levels of ActA protein in lysates from cells treated with type I IFN (Fig. 4b, c). We also examined InlB, a bacterial surface protein required for invasion of host cells ${ }^{40}$. Levels of InlB dropped in the TKO $\mathrm{Lm}$ strain compared to WT bacteria, consistent with degradation of InlB by phagosomal proteases. However, levels of InlB were unchanged in IFN $\beta$ treated BMDMs infected with WT and TKO Lm (Fig. 4b, c). These findings indicate that type I IFN selectively modulates phagosomal proteolysis of bacterial surface-associated proteins. 
a

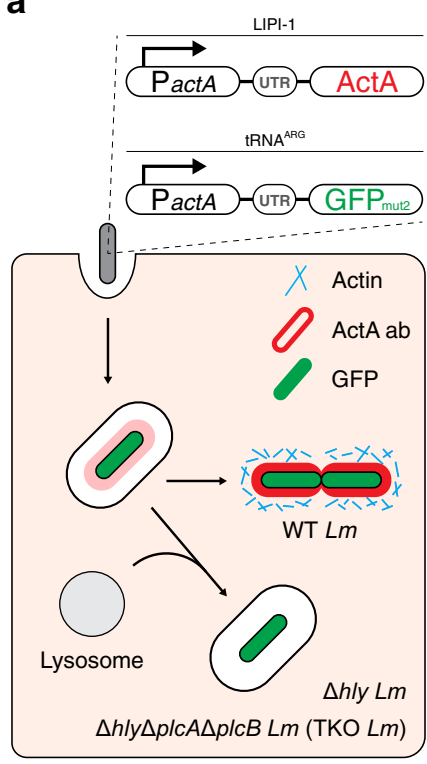

b

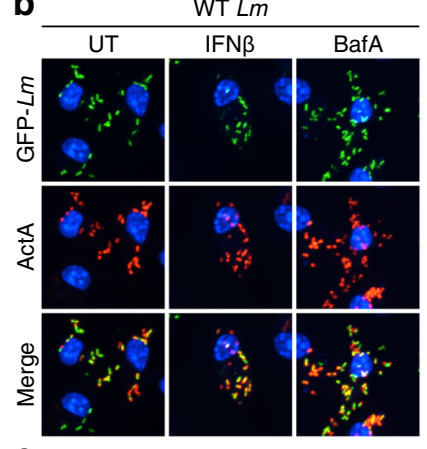

C

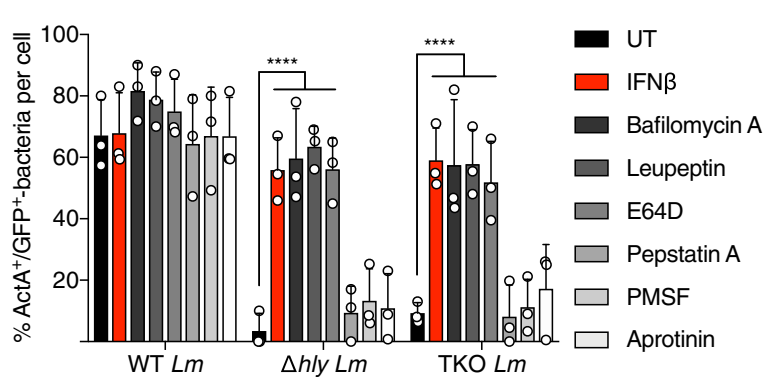

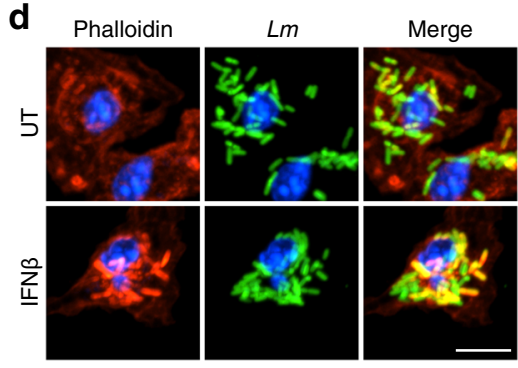
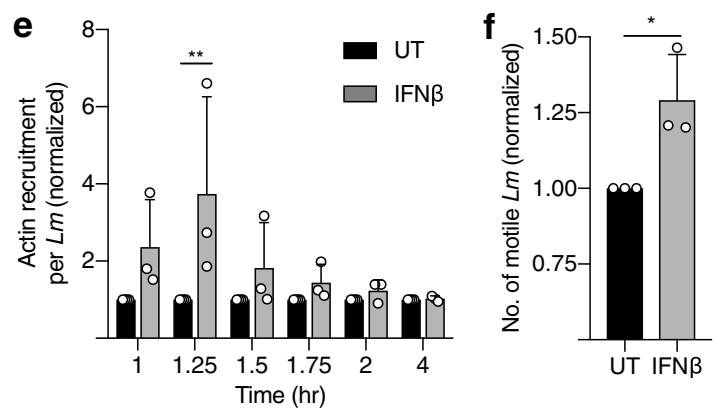

Fig. 3 Type I IFN suppresses ActA degradation in phagosomes and promotes $\mathbf{L m}$ actin recruitment. a Schematic of GFP reporter $L m$ strains developed to measure act $A$ transcription activation. Representative images (b) and quantification (c) of actA:GFP reporter expression and ActA degradation of BMDMs infected for $4 \mathrm{~h}$ with indicated $\mathrm{Lm}$ strains measured by GFP fluorescence and antibody staining, with or without treatment of IFN $\beta$, bafilomycin A or protease inhibitors. Representative images at $1.25 \mathrm{hr}$ p.i. (d) and quantification (e) of WT Lm actin recruitment, measured by phalloidin staining, after phagosome escape over $4 \mathrm{~h}$ time course. $\mathbf{f}$ Measurement of WT Lm actin-based motility with phalloidin-positive comet tails $4 \mathrm{~h}$ p.i. Data shown are means $\pm s$.d. for $n=3$ independent experiments. $P$ value was calculated using (c, e) two-way ANOVA and (f) two-tailed unpaired $t$-test. ${ }^{\star} P=0.0285,{ }^{\star \star} P=0.001,{ }^{\star \star \star \star} P<0.0001$. Scale bars, $11 \mu \mathrm{m}$.

We also explored the impact of type I IFN on secreted LLO. For these studies, we used a triple mutant of $\mathrm{Lm}$ lacking other phagosome escape factors $(\triangle a c t A, \triangle p l c A$, and $\Delta p l c B)$ to decrease the efficiency of bacterial entry to the cytosol. Secreted LLO was isolated from the detergent soluble fraction of cell lysates isolated after $L m$ infection (Fig. 4a). In IFN $\beta$-treated BMDMs, secreted LLO was elevated in the $\triangle a c t A p l c A p l c B$ Lm strain at 1,2 and $4 \mathrm{~h}$ p.i. (Fig. 4d, e). We also observed higher levels of secreted LLO at $4 \mathrm{~h}$ p.i. by WT $\mathrm{Lm}$. We conclude that type I IFN suppresses phagosomal proteolysis of $L m$ virulence factors required for phagosome escape.

Since ActA and LLO contribute to phagosome escape ${ }^{22,35}$, we examined the impact of type I IFN treatment on this process. IFN $\beta$ treatment led to an increase in actin recruitment to WT $L m$ during the early stages of bacterial spread (Fig. 3d, e), concomitant with a higher number of motile $L m$ with actin comet tails at $4 \mathrm{~h}$ p.i. (Fig. 3f). These findings are consistent with our prior study showing that type I IFN promotes $\mathrm{Lm}$ cell-to-cell spread $^{20}$, possibly through a positive feedback mechanism between actin polymerization and comet tail formation previously described ${ }^{4}$. Together, these results reveal that type I IFNmediated suppression of phagosomal proteolysis can promote the activity of $\mathrm{Lm}$ virulence factors that regulate intracellular infection and dissemination of these bacteria.
IFITM3 promotes $\mathbf{L} \boldsymbol{m}$ cell-to-cell spread. To enter the cytosol during infection of host cells, some viruses exploit proteases during the viral fusion process. For example, severe acute respiratory syndrome coronavirus (SARS-CoV) and SARS-CoV-2 utilizes host type II transmembrane serine proteases and cathepsin L to infect cells expressing its ACE2 receptor ${ }^{42-44}$. Conformational changes in the viral $S$ glycoprotein mediated by proteolysis are thought to enable viral fusion. Similarly, endosomal proteolysis is required for infection by Ebola virus, influenza A and rotaviruses ${ }^{45-47}$.

Interferon-induced transmembrane protein 3 (IFITM3) is an antiviral ISG in mice and humans that localizes to cell surface and endocytic compartments in most cell types during type I IFN activation ${ }^{48-50}$. Consistent with this notion, we observed IFITM3 to be the only fusion and trafficking-associated protein strongly localizing to both phagosomes and lysosomes in IFN $\beta$-treated BMDMs (Fig. 2a). IFITM3 mediates viral resistance by blocking fusion between virus and host membranes ${ }^{51}$, and by inhibiting viral envelope processing and capsid protein proteolysis ${ }^{52,53}$. IFITM3 is also localized to bacteria-containing phagosomes during Legionella pneumophila infection ${ }^{54}$. Based on these findings we evaluated whether IFITM3 contributes to type I IFN-mediated changes in phagocyte function during $L m$ infection. 
a

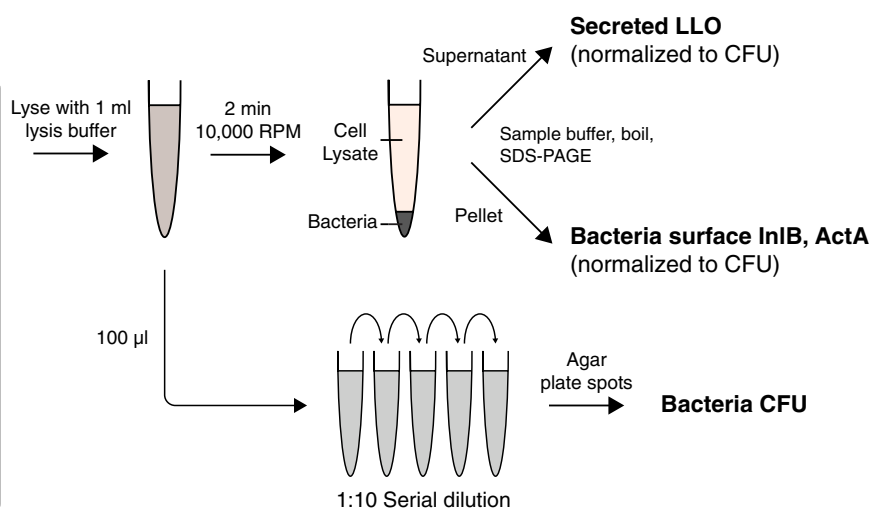

b

$\frac{\text { WT } L m}{\text { UT IFN } \beta} \frac{\text { TKO } L m}{U T}$

d $\frac{\text { WT } L m}{\text { UT IFN } \beta} \frac{\Delta \operatorname{actAplcAplcB} L m}{\text { UT IFN } \beta}$
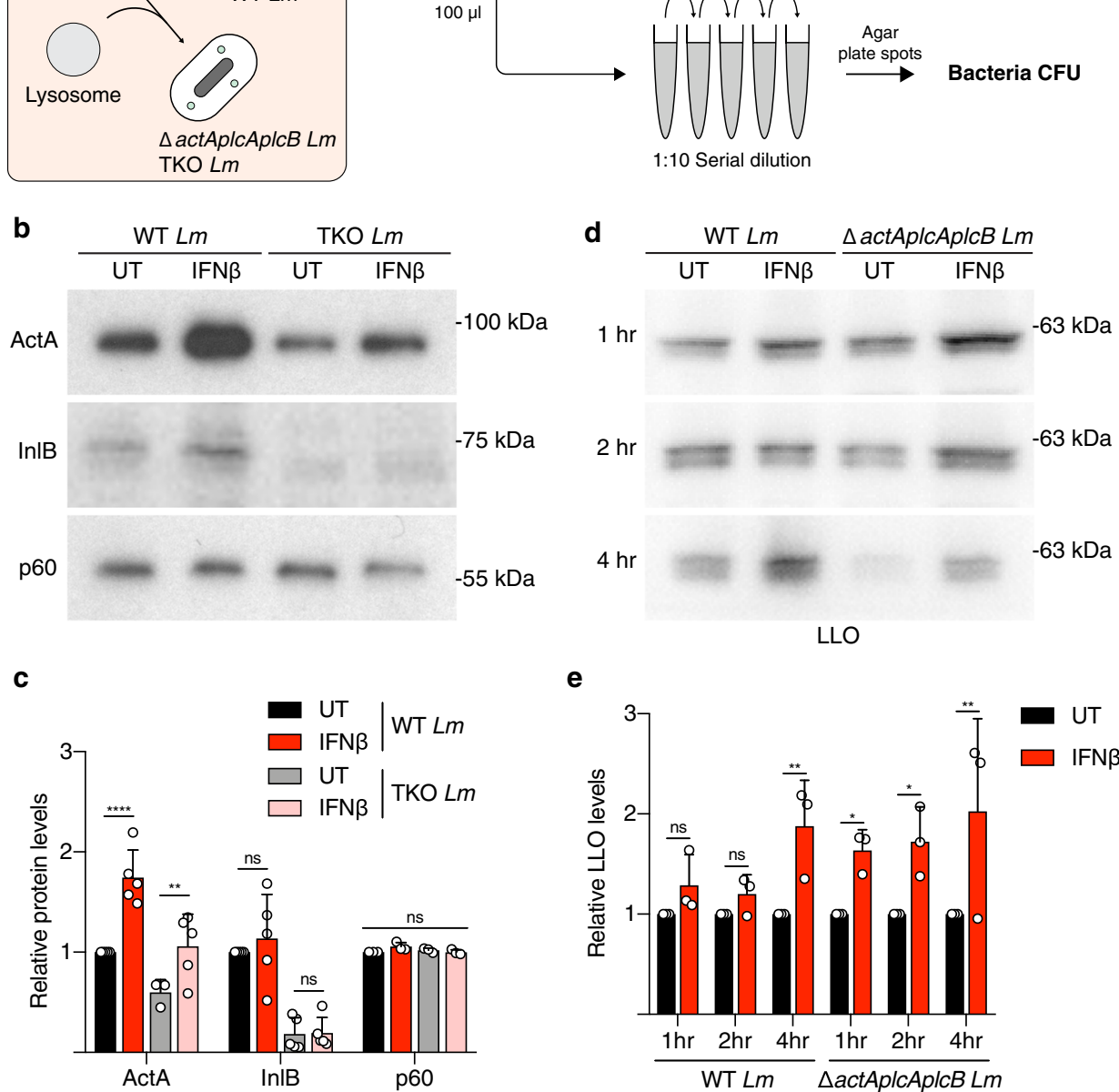

Fig. 4 Type I IFN suppresses $\mathbf{L m}$ virulence factor degradation in phagosomes. a Schematic of experimental protocol detailing methods to obtain secreted and bacterial lysate fractions from BMDMs infected with indicated $L m$ strains. Representative images (b) and quantification (c) of ActA and InIB on the bacteria surface measured by western blot in BMDMs infected with indicated $\mathrm{Lm}$ strains $4 \mathrm{~h}$ p.i. Cell wall-associated protein p60 used as loading control. ${ }^{\star \star} P=0.002,{ }^{\star \star \star \star} P<0.0001$. Representative images (d) and quantification (e) of LLO measured by western blot in BMDMs infected with indicated $L m$ strains over $4 \mathrm{~h} .{ }^{\star} P<0.0295,{ }^{\star} \times P<0.0039$. Data shown are means \pm standard deviation (s.d.) for at least $n=3$ independent experiments. $P$ value was calculated using two-way ANOVA. ns not significant, $P>0.1301$. Source data are provided as a Source Data file.

BMDMs were isolated from Ifitm $3^{-1-}$ mice (hereafter IFITM3 $\mathrm{KO})$ and infected with phagosome-confined bacteria. We observed a reduced bacterial load over time for both $\Delta h l y \mathrm{Lm}$ and E. coli in untreated BMDMs from WT and IFITM3 KO mice, consistent with antibacterial activity in macrophage phagosomes (Fig. 5a, b). However, the type I IFN-mediated defect in antibacterial activity was not observed in IFITM3 KO BMDMs. Thus, IFITM3 contributes to suppression of killing of phagosome-confined bacteria following type I IFN treatment.

We also examined the role of IFITM3 in suppressing phagosomal proteolysis. Type I IFN-mediated accumulation of ActA on the surface of phagosome-confined actA:GFP reporter Lm ( $\Delta h l y$ and TKO) was lost in IFITM3 KO BMDMs (Fig. 5c, d). Similarly, type I IFN-mediated accumulation of secreted LLO in WT and $\triangle a c t A p l c A p l c B$ Lm-infected BMDMs was lost in IFITM3
KO cells (Supplementary Fig. 4a-c). Next, we looked at early proteolysis dynamics in live cells using DQ-BSA labeled beads. We found that the decrease in proteolysis of IFN $\beta$-treated BMDMs was not affected by the loss of IFITM3 (Supplementary Fig. 5a, b). We also performed proteomic analysis of phagosomes isolated from IFITM3 KO BMDMs after $4 \mathrm{~h}$. Loss of IFITM3 was sufficient to revert some of the IFN $\beta$-mediated alterations to proteins responsible for hydrolytic activity and fusion and trafficking events (Fig. 2b). Lysosomal protein content was mostly unaffected by IFN $\beta$ treatment (Fig. 2b), suggesting a decrease in proteolysis only in the phagosome. Finally, we quantified and found a type I IFN-mediated increase in actin recruitment in WT Lm-infected BMDMs, an effect that was lost in IFITM3 KO cells (Fig. 5e, f). Together, these findings indicate 

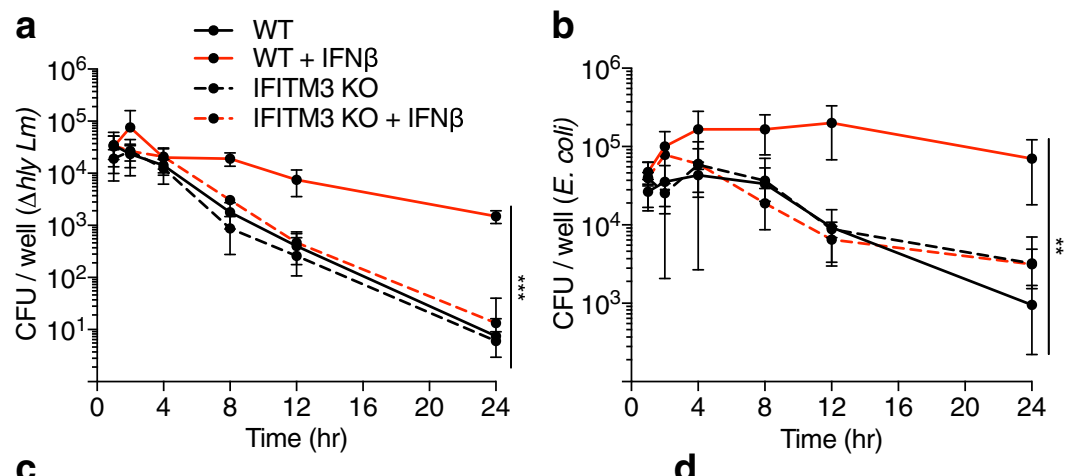

C
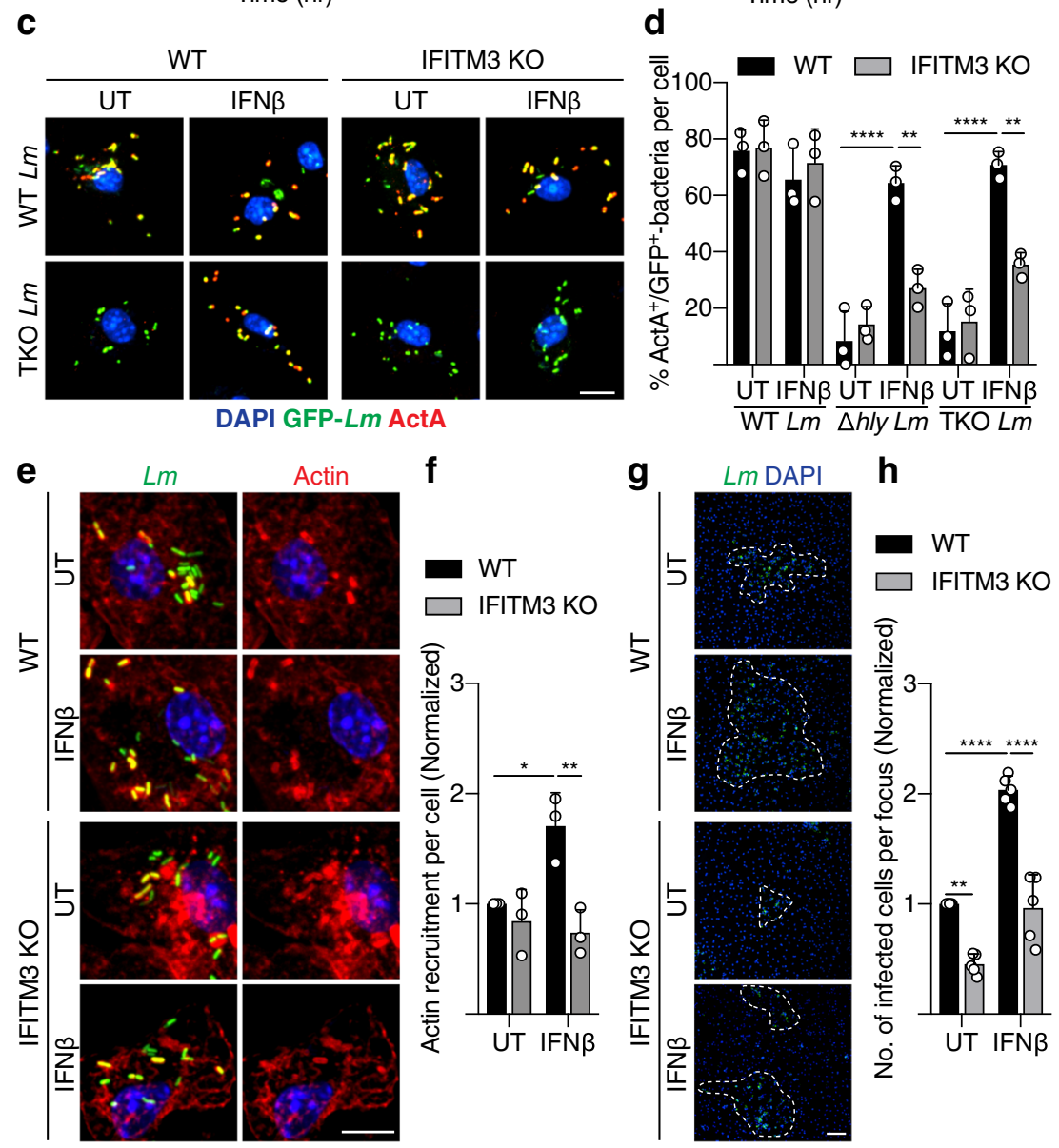

Fig. 5 IFITM3 suppresses antibacterial activity in phagosomes and promotes $\mathbf{L m}$ cell-to-cell spread. Time course of bacteria killing over $24 \mathrm{~h}$ of (a) $\Delta h l y$ Lm or (b) E. coli infected WT and IFITM3 KO BMDMs with or without IFN $\beta$. Representative images (c) and quantification (d) of ActA degradation of IFITM3 WT and KO BMDMs infected for $4 \mathrm{~h}$ with indicated actA:GFP reporter $\mathrm{Lm}$ strains measured by antibody staining of surface Act $\mathrm{A}$ under treatment with or without IFN $\beta$. Scale bar, $11 \mu \mathrm{m}$. Representative images (e) and quantification (f) of WT Lm actin recruitment after phagosome escape at $1.25 \mathrm{~h}$ p.i. in IFITM3 WT and KO BMDMs treated with or without IFN $\beta$. Scale bar, $11 \mu \mathrm{m}$. Representative images (g) and quantification (h) of an infection focus assay to measure WT Lm cell-to-cell spread. WT and IFITM3 KO BMDMs were treated with or without IFN $\beta$ and infected for $18 \mathrm{~h}$. Dotted lines delineate edge of the infection foci. Scale bar, $70 \mu \mathrm{m}$. Data shown are means \pm s.d. for at least $n=3$ independent experiments. $P$ value was calculated using two-way ANOVA. ${ }^{\star} P$ $=0.0242,{ }^{\star \star} P<0.0041,{ }^{\star \star \star} P=0.001,{ }^{\star \star \star \star} P<0.0001$.

that IFITM3 selectively modulates phagosomal proteolysis and bacteria phagosome escape following type I IFN treatment.

Next, we examined the impact of IFITM3 on cell-to-cell spread of WT $L m$ by performing an infection focus assay ${ }^{7}$. We show that IFITM3 KO BMDMs displayed a decrease in $L m$ spread (Fig. 5g, h). We also found that IFN $\beta$-treated BMDMs displayed increased Lm cell-to-cell spread in an IFITM3-dependent manner. The ability of $L m$ to grow in the cytosol was unaffected by IFN $\beta$ or the loss of IFITM3 (Supplementary Fig. 5c). These results suggest that expression of IFITM3 plays a significant role in promoting $\mathrm{Lm}$ cell-to-cell spread in BMDMs.

IFITM3 promotes $\mathbf{L m}$ infection in vivo. Next, we examined the impact of IFITM3 on $L m$ infection in vivo. WT and IFITM3 KO mice were inoculated by tail vein injection with WT $\mathrm{Lm}$ to examine 10-day survival, as well as to obtain tissue homogenates from the liver, spleen, and brain to measure bacterial load after $72 \mathrm{~h}$. IFITM3 KO mice were protected against $L m$ infection, with 

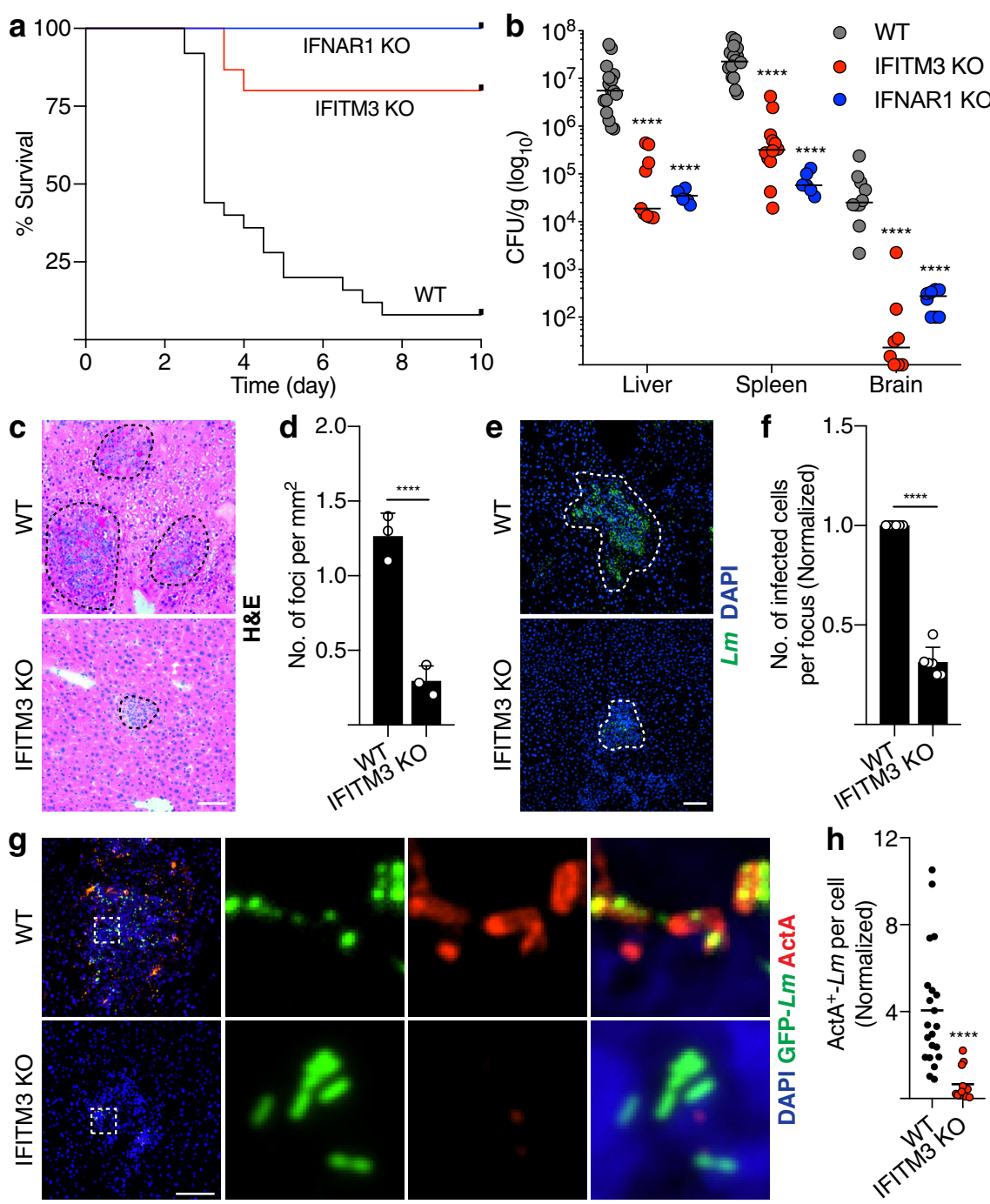

Fig. 6 IFITM3 promotes $\mathbf{L m}$ infection in vivo. a 10-day survival rate of WT $(n=25)$, IFITM3 $(n=15)$, and IFNAR1 KO $(n=10)$ mice infected with WT Lm plotted using Kaplan-Meier function. b Bacteria load of liver, spleen and brain of WT ( $n \geq 10)$, IFITM3 ( $n \geq 8)$, and IFNAR1 KO ( $n \geq 6)$ mice infected with Lm over $72 \mathrm{~h}$. Representative images $(\mathbf{c}, \mathbf{e})$ and quantification $(\mathbf{d}, \mathbf{f})$ of infection foci taken from the liver of mice $(n=3) 72 \mathrm{~h}$ p.i. Tissues were stained with H\&E (c, d) or Lm and DAPI (e, f). Dotted lines delineate edge of the infection foci. Representative images ( $(\mathbf{g})$ and quantification (h) of cell surface ActA expression by $L m$ in liver taken from mice $(n=3) 72 \mathrm{~h}$ p.i. Data shown are medians $(\mathbf{b}, \mathbf{h})$ or means $\pm \mathrm{s}$.d. (d, $\mathbf{f})$. $P$ value was calculated using two-tailed Mann-Whitney test $(\mathbf{b}, \mathbf{f})$ and two-tailed unpaired $t$-test $(\mathbf{d}, \mathbf{f}) .{ }^{\star \star \star \star} P<0.0001$. Scale bars, $70 \mu \mathrm{m}$.

increased survival rates after 10 days compared to WT mice (Fig. 6a). IFITM3 KO mice also displayed a significant decrease in bacteria harvested from the liver, spleen and brain compared to control animals (Fig. 6b). Consistent with this, liver sections from IFITM3 KO mice revealed a decrease in total number of infection foci per square mm of liver tissue (Fig. $6 c$, d), as well as a decrease in the number of infected cells per infection focus (Fig. 6e, f), compared to WT mice. Similar changes were observed in IFNAR1 $\mathrm{KO}$ mice (Fig. 6a, b), consistent with previous findings ${ }^{14}$. We also found higher numbers of ActA-positive $\mathrm{Lm}$ in WT compared to IFITM3 KO livers (Fig. 6g, h), consistent with a decrease in proteolysis of this virulence factor seen in vitro. Together, this data suggests that IFITM3 is a key downstream target of type I IFN signaling that $\mathrm{Lm}$ exploits to promote its dissemination.

IFITM3 deficiency has previously been shown to display higher levels of inflammatory cytokines during a viral infection ${ }^{55}$. Therefore, we examined the role of IFITM3 in cytokine production and immune cell recruitment during $\mathrm{Lm}$ infection. We found no change in levels of IFN $\beta$ production in $L m$-infected WT and IFITM3 KO mice and BMDMs (Fig. 7a, b). In IFITM3
KO mice we observed an increase in expression of proinflammatory cytokines such as TNFa, IL-4 and IL-12, and reduced expression of the anti-inflammatory cytokine IL-10 (Fig. 7c-f). This effect was not due to a change in IFN gamma receptor expression (Fig. 7g). IFITM3 KO mice also displayed higher levels of lymphocyte and macrophage recruitment to sites of infection, as judged by CD3e and F4/80 staining of infected tissues, respectively (Fig. $7 \mathrm{~h}-\mathrm{j}$ ). Thus, IFITM3 also promotes systemic infection of $\mathrm{Lm}$ in mice by suppressing immune cell recruitment and pro-inflammatory cytokine signaling.

\section{Discussion}

In this study, we show that type I IFN can suppress the antibacterial activities of phagocytes through the actions of IFITM3. This ISG has previously been shown to selectively modulate the activity of gamma-secretase, a protease complex ${ }^{56}$, playing an important part in regulating protease activity within the endosomal pathway. We show that IFITM3 can selectively suppress proteolysis of some phagosomal cargoes such as ActA and LLO, leading to increased $\mathrm{Lm}$ cell-to-cell spread (Fig. 8). Our findings 

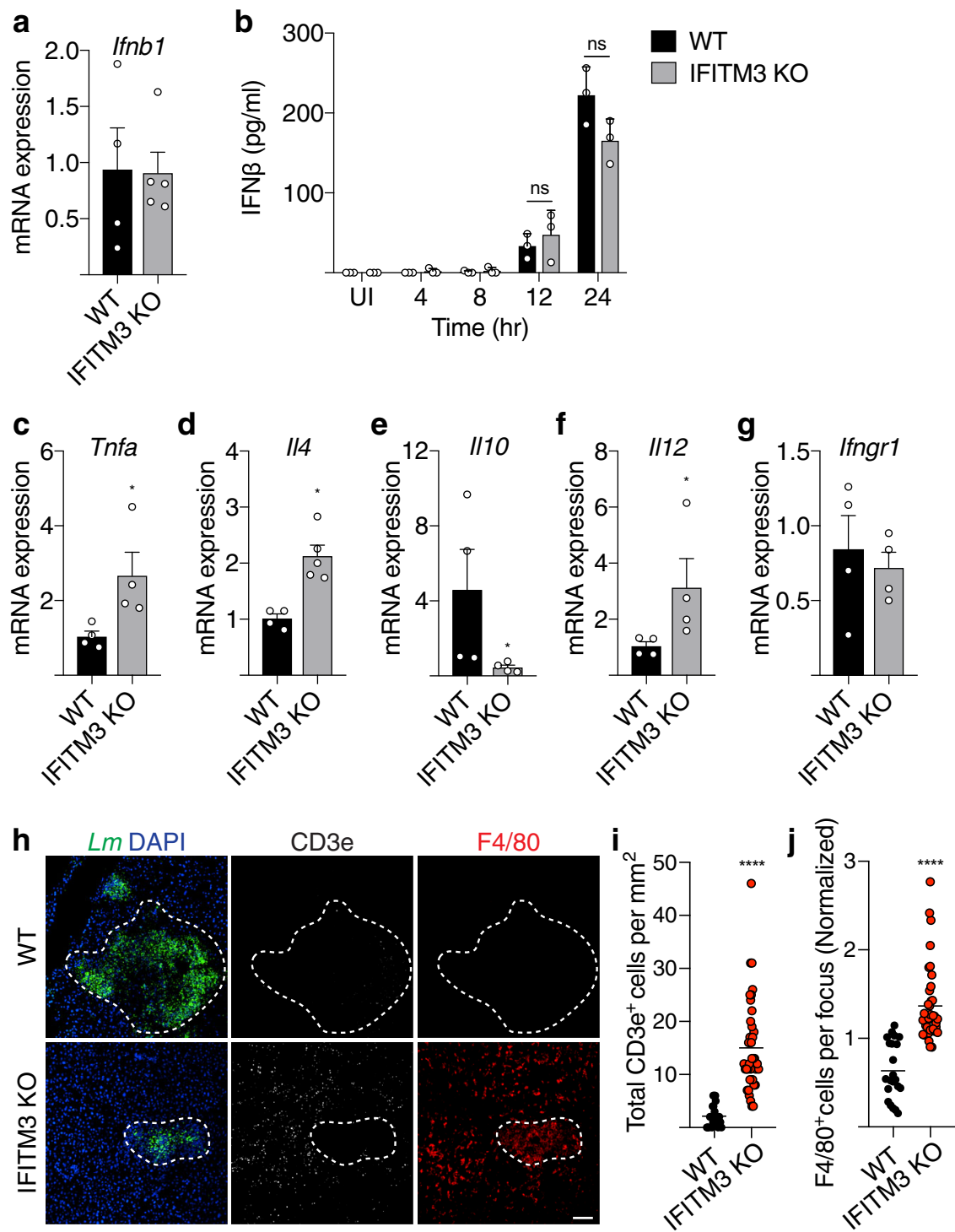

Fig. 7 IFITM3 modulates cytokine signaling and immune cell recruitment. a mRNA expression of IFN $\beta$ from $L m$-infected liver of mice $(n \geq 4) 72 \mathrm{~h}$ p.i. b IFN $\beta$ production measured by ELISA of WT and IFITM3 KO BMDMs infected with $L m$ over $24 \mathrm{~h}$. UI uninfected. c-g mRNA expression of indicated cytokines from $L m$-infected liver of mice $(n \geq 4) 72 \mathrm{~h}$ p.i. Representative images (h) and quantification of (i) lymphocyte recruitment (CD3e) and (j) macrophage recruitment (F/480) of liver taken from mice $(n=3) 72 \mathrm{~h}$ p.i. Dotted lines delineate edge of the infection foci. Data shown are means (a-g) or medians $(\mathbf{i}, \mathbf{j}) \pm$ s.d. for at least $n=3$ independent experiments. $P$ value was calculated using two-tailed unpaired $t$-test (a-g) and two-tailed Mann-Whitney test $(\mathbf{i}, \mathbf{j}) .{ }^{\star} P<0.05,{ }^{\star \star \star \star} P<0.0001$. ns not significant, $P>0.055$. Scale bar, $70 \mu \mathrm{m}$.

provide important insight into the expression and function of ActA, which was previously thought to be expressed exclusively in the cytosol 37,38 , while also revealing the impact of IFITM3 expression on this virulence factor in vivo during $L m$ infection.

IFITM3 likely benefits $\mathrm{Lm}$ infection through multiple mechanisms. The bacteria are known to secrete LLO during $\mathrm{Lm}$ protrusion formation to induce damage at the plasma membrane and promote cell-to-cell spread using a mechanism that resembles efferocytosis ${ }^{7}$. Membrane repair at the site of protrusion is key in preventing bacterial cell-to-cell spread by limiting exofacial PS exposure $^{57}$. In the presence of type I IFN, macrophages and lymphocytes are sensitized to membrane damage mediated by LLO toxin by mechanisms that remain unclear ${ }^{58}$. It is possible that IFITM3 may be playing a separate role in promoting $L m$ cell-tocell spread by suppressing lysosome-mediated membrane repair.
Our results also reveal a role of IFITM3 in regulating immune cell recruitment and cytokine signaling in infected tissues, though the mechanisms for this remain unclear. Type I IFN was shown to induce T-lymphocyte apoptosis through the actions of LLO $^{14,59,60}$. Whether IFITM3 affects T-lymphocyte apoptosis to promote $\mathrm{Lm}$ infection is an important question for future studies. It is noteworthy that IFITM3 was recently shown to modulate B cell activation through promoting PI3-kinase signaling ${ }^{50}$. IFITM3 has also been implicated in a wide range of diseases such as cancer metastasis, tuberculosis susceptibility, and ulcerative colitis $^{61-63}$. Our findings are consistent with an emerging view that IFITM3 is involved in many biological processes, beyond its established role in antiviral immunity.

The importance of type I IFN during bacterial infections is highlighted by its significant role in many other host-pathogen 


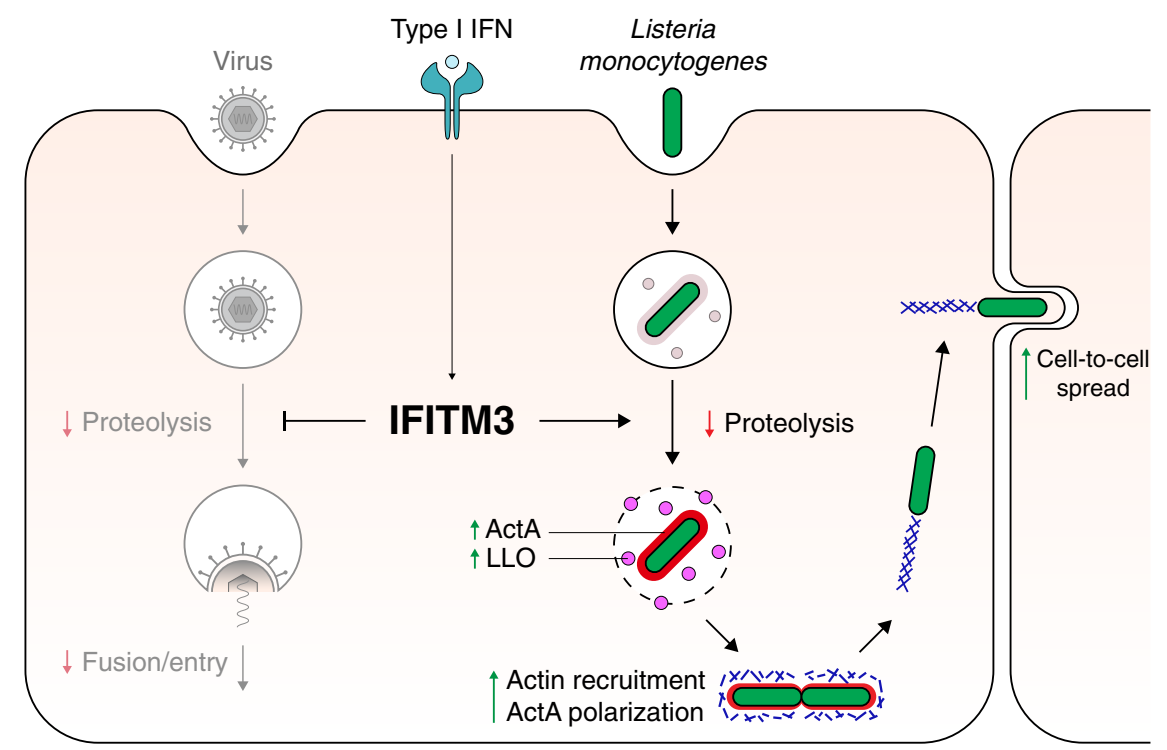

Fig. 8 Model of type I IFN-mediated effects of IFITM3. During viral infection, IFITM3 decreases proteolysis of virus capsid proteins, and restricts fusion and entry into the cytosol. During Lm infection, IFITM3-mediated suppression of proteolysis leads to increased LLO and ActA in the phagosome. After entering the cytosol, increased actin recruitment subsequently leads to an increase in cell-to-cell spread. Arrows indicate decrease (red) or increase (green) by IFITM3.

interactions ${ }^{64}$. Failure to clear Streptococcus pneumoniae and Staphylococcus aureus in alveolar macrophages after a viral infection is attributed to IFN-induced alteration of the innate and adaptive immune response ${ }^{65,66}$. Type I IFN and IFITM3 have also been found to promote Salmonella enterica serovar Typhimurium infection in epithelial cells by increasing lysosomal acidification and proteolysis ${ }^{67}$. However, this effect may be celltype specific as mice infected with $S$. Typhimurium and other non-cytosolic pathogens are unaffected by a loss of IFITM3 ${ }^{68}$. Consistent with this notion, we found that type I IFN treatment does not promote $\mathrm{Lm}$ spread in non-phagocytic cells such as fibroblasts and Caco-2, whereas spread is decreased in hepatocytes (Supplementary Fig. 6a-f). In future studies, it will be important to determine if other bacterial pathogens that undergo cytosolic growth and cell-to-cell spread can also exploit IFITM3 during infection in phagocytes. Since IFITM3 appears to drive much of the deleterious effects of type I IFN on the host to benefit $\mathrm{Lm}$ during infection, it represents a possible therapeutic target for the treatment of this class of bacterial pathogens.

\section{Methods}

Bacterial strains. Listeria monocytogenes $(\mathrm{Lm})$ were grown in brain-heart infusion (BHI) broth and the following strains were used: 10403S (WT), $10403 \mathrm{~S}$ (dasherGFP-WT), DP-L2161 ( $\Delta h l y)$, DH-L1137 (GFP- $\Delta h l y)$, DP-L2319 $(\Delta h l y \Delta p l c A \Delta p l c B, \mathrm{TKO})$, and DH-1458 ( $\triangle a c t A \Delta p l c A \Delta p l c B)$. Escherichia coli were grown in lysogeny broth (LB) and the following strains were used: DH10B (WT) and $\mathrm{DH} 10 \mathrm{~B}$ (RFP). E. coli was cultured overnight, diluted to an $\mathrm{OD}_{600}$ of 1.0 and opsonized with IgG (Meridian) for $30 \mathrm{~min}$ at $37^{\circ} \mathrm{C}$ prior to infection.

The following reporter strains of $L m$ expressing GFP under the actA promoter were used: DH-L1245 (WT), DH-L2011 ( $\Delta h l y)$ and DH-L2012 $(\Delta h l y \Delta p l c A \Delta p l c B$, TKO). To generate the reporter stains, the reporter construct $\mathrm{P}_{\text {act }}$-actA UTR$g f p_{\text {mut } 2}$ was generated by overlap extension PCR ${ }^{69}$. Primer 432 (CGCGGCCGGTG GTATCCCGAATAAAGCAGCC, annealing 262 bp upstream of the actA transcriptional start site) and primer 434 (GAAAAGTTCTTCTCCTTTACTC ATTTATACTCCCTCCTCGTGATAC) were used to PCR amplify the actA promoter and UTR from the $\mathrm{Lm}$ chromosome. Primers 435 (GTATCACGAGGA GGGAGTATAAATGAGTAAAGGAGAAGAACTTTTC) and 221 (AAGTCGA CTTATTTGTATAGTTCATCCATGCCATG) were used to PCR amplify the $g f p_{\text {mut2 }}$ gene from plasmid pDH-1038 ${ }^{70}$. The two PCR products were gel purified using a QIAquick gel extraction kit (Qiagen), and the purified products were then used as templates for splicing by overlap extension PCR ${ }^{69}$ along with primers 432 and 221. The resulting $\mathrm{P}_{a c t A}-a c t A$ UTR- $g f p_{\text {mut2 }}$ fragment was gel purified, digested with EagI and SalI, and ligated into pPL3 digested with the same restriction enzymes. The ligation was then dialyzed and electroporated into E. coli XL1-Blue to generate strain DH-E1244. The resulting plasmid (pDH-1244) was also electroporated into Lm 10403 S to yield DH-L1245, Lm DP-L2161 to yield DH L2011, and Lm DP-L2319 to yield DH-L2012. Antibiotics were used at the following concentrations: chloramphenicol $20 \mu \mathrm{g} \mathrm{ml}^{-1}$ for selection of pPL3derived plasmids in E. coli and chloramphenicol $7.5 \mu \mathrm{g} \mathrm{ml}^{-1}$ for selection of singlecopy pPL3-derived plasmids integrated at the tRNA ${ }^{A R G}$ locus in $L m$. All primers used are listed in Supplementary Table 1.

Animals. B6.129S2-Ifnar1 ${ }^{-1-}$ (IFNAR1 KO; Jackson stock \#32045-JAX) and B6 (Cg)-Tyr ${ }^{-2 J}$-Ifitm $3^{-I-}$ (IFTIM3 KO; obtained from M. Diamond, backcrossed $>10$ generations with $\mathrm{C} 57 \mathrm{BL} / 6 \mathrm{~J}$ at Washington University at St. Louis ${ }^{71}$ ) mice were bred in-house at The Hospital for Sick Children Animal Care Facility. C57BL/6J (stock \#000664) and B6(Cg)-Tyr ${ }^{c-2 J}$ (stock \#000058) mice, originally from The Jackson Laboratory, were also bred in-house and used as controls. All experiments were performed with 6-12-week-old sex- and age-matched mice that were maintained on a $12 \mathrm{~h}$ light-dark cycle, with food and water available ad libitum. All animals were bred separately and housed in specific pathogen-free barrier rooms kept at $22-26^{\circ} \mathrm{C}$ and $45-55 \%$ humidity. Experiments were not blinded, and mice were not randomized. All experiments described in this study were carried out in accordance with the Guide for the Humane Use and Care of Laboratory Animals and ethical approval was obtained from The Hospital for Sick Children's Animal Care Committee (AUP \#52111).

Antibodies, constructs, and reagents. Primary antibodies (1:100) used for immunofluorescence were rabbit anti- $\mathrm{Lm}$ (\#B223021 from BD Biosciences), rabbitanti-E. coli (\#ab20640 from Abcam), rabbit-anti-ActA ${ }^{72}$ (gift from P. Lauer, Aduro BioTech), rabbit-anti-InlB (gift from M. Loessner, ETH Zurich), mouse-anti-p60 (\#NBP2-80120 from Novus), rabbit-anti-LLO (\#ab200538 from Abcam), rat-antiF4/80 (\#ab16911 from Abcam), biotin-anti-CD3e (\#13-0031-85 from ThermoFisher), and rat anti-mouse LAMP1 (1D4B was deposited to the Developmental Studies Hybridoma Bank by J.T. August). Primary antibodies (1:1000) used for western blotting were rabbit-anti-ActA, rabbit-anti-InlB (gift from M. Loessner ETH Zurich), rabbit-anti-LLO (\#ab200538 from Abcam). Alexa Fluor-568/647 Phalloidin and all fluorescent secondary antibodies (Alexa Fluor conjugates) were from Invitrogen. IFN $\beta$ (\#8234-MB-010; $2400 \mathrm{IU} \mathrm{ml}^{-1}$ ) was from R\&D Systems and IFN $\gamma$ (\#315-05; $100 \mathrm{U} \mathrm{ml}^{-1}$ ) was from PeproTech. 4,6-Diamidino-2-phenylindole (DAPI) (\#D1306 from Invitrogen) was used at 1:2,500 dilution to stain the nuclei where indicated. Green DQ-BSA (\#D12050; $10 \mu \mathrm{g} \mathrm{ml}^{-1}$ ) and Oregon Green Dextran (\#D7173; $40 \mu \mathrm{g} \mathrm{ml}^{-1}$ ) was from ThermoFisher. Cresyl violet (\#C5042;1 $\left.\mu \mathrm{M}\right)$ and FITC-dextran (\#FD10S) was from Sigma. Bafilomycin A (\#11038; $100 \mathrm{nM}$ ) was from Cayman Chemical. The following protease inhibitors were from BioShop: Leupeptin (\#LEU001.25; $200 \mu \mathrm{M}$ ), PMSF (\#PMSF123; $1 \mathrm{mM}$ ), Aprotinin (\#APR600.100; $1.5 \mu \mathrm{M}$ ), Pepstatin A (\#PEP605.10; $10 \mu \mathrm{M})$. E64D (\#E8640; 100 $\mu \mathrm{M})$ was from Sigma.

Cell culture and macrophage generation. RAW264.7 and J774 macrophages, AML12 hepatocytes, mouse embryonic fibroblasts (MEFs), and Caco-2 cells were cultured in DMEM (Hyclone) supplemented with $10 \%$ heat-inactivated FBS 
(Wisent) without antibiotics at $37^{\circ} \mathrm{C}$ and $5 \% \mathrm{CO}_{2}$. All cells used were authenticated and tested negative for mycoplasma by The Hospital for Sick Children Biobank and the Mycoplasma Plus PCR kit (Aligent Technologies \#302008). Primary macrophages were obtained from mice housed in The Hospital for Sick Children Animal Care Facility. Mice were euthanized by $\mathrm{CO}_{2}$ inhalation. Mouse BMDMs were obtained from the dissected femurs and tibias of 6-8-week-old male C57BL/6 mice. Cells were washed with growth medium and plated on $70 \mathrm{~cm}^{2}$ Petri dishes (ThermoFisher \#FB0875712). Medium was replaced every 2-3 days and after 7 days cells were used for experiments. Cells were maintained in high-glucose RPMI-1640 medium (Wisent \#350-025-CL) containing 10\% heat-inactivated FBS (Wisent), $1 \%$ sodium pyruvate (Wisent), $1 \%$ non-essential amino acids (Wisent), $0.5 \%$ 2-mercaptoethanol (Gibco), 1\% penicillin and streptomycin (Invitrogen), and 10\% L929-conditioned media while differentiating, and in RPMI and 10\% FBS subsequently. L929-conditioned media was generated by growing a confluent layer of L929 cells in $175 \mathrm{~cm}^{2}$ flasks in DMEM supplemented with $10 \%$ heat-inactivated FBS. When cells reached confluency, growth medium was replaced with DMEM alone. After 7 days, supernatant was collected, centrifuged, filtered and stored at $-20^{\circ} \mathrm{C}$.

Immunofluorescence. Immunostaining was conducted as previously described ${ }^{57}$. Briefly, cells were fixed with $2.5 \%$ paraformaldehyde (PFA) for $10 \mathrm{~min}$ at $37^{\circ} \mathrm{C}$, permeabilized and blocked in PBS+ (Wisent) containing $0.2 \%$ saponin (Calbiochem) and $10 \%$ normal goat serum (SS-PBS) for 30 min. Subsequently, cells were incubated for $1 \mathrm{~h}$ with primary antibodies in SS-PBS. Cells were washed three times with PBS+ and incubated with secondary Alexa Fluor conjugated antibodies in SSPBS for $1 \mathrm{~h}$. Cells were washed three times with PBS+, mounted in fluorescence mounting medium (Dako). Confocal microscopy images were imported into Adobe Photoshop and assembled in Adobe Illustrator for labeling.

Western blotting. Samples were normalized to bacterial CFU and subjected to SDS-PAGE using 12\% polyacrylamide gels (Invitrogen \#XP00125BOX) and transferred onto PVDF membranes (BioRad \#1620177). Membranes were blocked using 5\% non-fat dried milk diluted in TBS-Tween for $1 \mathrm{~h}$. Primary and HRPsecondary antibodies were diluted at 1:1000 and 1:5000 respectively. Membranes were detected using West Pico PLUS chemiluminescent substrate (ThermoFisher \# 34580) on a Bio-Rad ChemiDoc system. Densitometry was performed using ImageJ software. All uncropped and unprocessed scans of blots can be found in the Source Data File.

Bacteria killing assay. WT, IFNAR1 KO, and IFITM3 KO BMDMs were plated at $3 \times 10^{5}$ cells per well in 24 -well tissue culture plates and treated with $2400 \mathrm{IU} \mathrm{ml}^{-1}$ IFN $\beta 24 \mathrm{~h}$ before infection. Cells were infected with $\Delta h l y L m$ or $E$. coli at an MOI of 1 or 1:200 respectively in RPMI. At 60 min p.i., all cells were washed three times with PBS+ and cultured in RPMI medium containing $10 \% \mathrm{FBS}, 50 \mu \mathrm{g} \mathrm{ml}^{-1}$ gentamicin (Wisent $\# 400-130-\mathrm{IG}$ ) and IFN $\beta$. At $1,2,4,8,12$, and $24 \mathrm{~h}$ p.i., cells were lysed with PBS+ containing $1 \%$ Triton X-100. Serial dilutions of the lysates were plated on LB- (E. coli) and BHI- ( $\Delta$ hly Lm) agar plates and incubated at $37^{\circ} \mathrm{C}$ for $16 \mathrm{~h}$ for subsequent quantification of intracellular colony forming units (CFUs).

Phagocytosis assay. BMDMs were plated at $3 \times 10^{5}$ cells per well in 24-well tissue culture plates and treated with $2400 \mathrm{IU} \mathrm{ml}^{-1}$ IFN $\beta 24 \mathrm{~h}$ before infection. Cells were infected with $\Delta h l y L m$ or E. coli at an MOI of 1 or 1:200 respectively in RPMI. At $60 \mathrm{~min}$ p.i., all cells were washed three times with PBS+ and cultured in RPMI medium containing $10 \% \mathrm{FBS}, 50 \mu \mathrm{g} \mathrm{ml}{ }^{-1}$ gentamicin and IFN $\beta$. At 5, 10, 15, 20, 25 and $30 \mathrm{~min}$ p.i., cells were fixed with $2.5 \%$ PFA. Differential antibody staining was carried out to stain extracellular bacteria before permeabilizing the cells to stain for total bacteria. Cells were imaged on a Quorum spinning disk confocal scan head (Leica DMI 6000 B inverted fluorescence microscope, Hamamatsu ORCA Flash 4 sCMOS and color camera) equipped with a $\times 63$ objective. Phagocytosis was measured by calculating internalized bacteria (total bacteria minus extracellular bacteria) over the time course using Volocity 6 software.

LAMP1 recruitment. BMDMs were plated at $3 \times 10^{5}$ cells per well in 24 -well tissue culture plates and treated with $2400 \mathrm{IU} \mathrm{ml}^{-1}$ IFN $\beta 24 \mathrm{~h}$ before infection. Cells were infected with $\Delta h l y L m$ or $E$. coli at an MOI of 1 or 1:200 respectively in RPMI. At 60 min p.i., all cells were washed three times with PBS+ and cultured in RPMI medium containing $10 \% \mathrm{FBS}, 50 \mu \mathrm{g} \mathrm{ml}^{-1}$ gentamicin and IFN $\beta$. At 1, 2, 4, 8, 12, 24 h p.i., cells were fixed with $2.5 \%$ PFA. Cells were stained with antibodies against Lm, E. coli and LAMP1. Cells were then imaged on a Quorum spinning disk confocal scan head equipped with a $\times 63$ objective. LAMP1 recruitment was measured by counting LAMP1-positive bacteria over the time course.

Cresyl violet assay. BMDMs were plated at $1 \times 10^{5}$ cells per well in $\mu$-Slide 8 Well IBIDI chambers (\#80826) and treated with $2400 \mathrm{IU} \mathrm{ml}^{-1}$ IFN $\beta 24 \mathrm{~h}$ before infection. Cells were infected with GFP- $\Delta h l y L m$ at an MOI of 5 in RPMI. At 60 min p.i., all cells were washed three times with PBS+ and cultured in RPMI medium containing $10 \%$ FBS, IFN $\beta$ and $50 \mu \mathrm{g} \mathrm{ml}^{-1}$ gentamicin. Cells were pulsed with cresyl violet $(1 \mu \mathrm{M})$ for 5 min to label acidified compartments ${ }^{73}$, and washed prior to imaging and replaced with RPMI medium containing $10 \%$ FBS and IFN $\beta$. $4 \mathrm{~h}$ p.i., cells were imaged live on a Quorum spinning disk confocal scan head (Leica DMI6000B inverted fluorescence microscope, Hamamatsu ImagEM $\times 2$ camera) equipped with a $\times 63$ objective. Cresyl violet-positive bacteria were quantified using Volocity 6 software.

Compartment fusion assay. BMDMs were plated at $1 \times 10^{5}$ cells per well in $\mu$ Slide 8 Well IBIDI chambers and treated with $2400 \mathrm{IU} \mathrm{ml}^{-1}$ IFN $\beta 24 \mathrm{~h}$ before treatment. 15 min prior to cresyl violet treatment, cells were pulsed with Oregon Green $10 \mathrm{kDa}$ dextran $\left(40 \mu \mathrm{g} \mathrm{ml}^{-1}\right)$ and washed. Cells were pulsed with cresyl violet $(1 \mu \mathrm{M})$ for $5 \mathrm{~min}$ and washed prior to imaging and replaced with RPMI medium containing $10 \%$ FBS and IFN $\beta$. Cells were imaged live using a spinning disk confocal microscope equipped with a $\times 63$ objective. Pearson correlation of dextran-positive and cresyl violet-positive compartment colocalization were quantified using Volocity 6 software.

Phagosome assay preparation. BMDMs were plated at $1.2 \times 10^{5}$ cells per well in 96 well $\mu$ Clear plates (Greiner Bio-One) and treated with $100 \mathrm{U} \mathrm{ml}^{-1}$ IFN $\gamma$ or $2400 \mathrm{IU} \mathrm{ml}^{-1} \mathrm{IFN} \beta 20 \mathrm{~h}$ prior to assay. Fluorescently labeled, IgG-coupled 3- $\mu \mathrm{m}$ silica particles were prepared and used for phagosomal lumenal characterization as previously detailed 74,75 . Measurements were performed in microplate format with the use of a FLUOstar Optima fluorescent plate reader (BMG Labtech) or Envision 2104 Multilabel Reader (PerkinElmar) at $37^{\circ} \mathrm{C}$. Immediately prior to the addition of experimental particles, growth medium was removed from cells and replaced with assay medium (DPBS containing $1 \mathrm{mM} \mathrm{CaCl}, 2.7 \mathrm{mM} \mathrm{KCl}, 0.5 \mathrm{mM} \mathrm{MgCl}_{2}, 5$ $\mathrm{mM}$ glucose, $0.1 \%$ calf skin gelatin, and $10 \mathrm{mM}$ HEPES). Particles were added to BMDMs at an MOI of 2-3 particles per cell.

Phagolysosomal fusion. BMDMs were prepared as above. To determine the rate of phagosome fusion with lysosomes, a FRET-based assay was used wherein the acceptor fluorophore (Alexa Fluor 594 hydrazide) was pulsed into lysosomes overnight prior to phagocytosis of the particle restricted donor fluorophore (Alexa Fluor $488 \mathrm{SE}$ ). The assay was carried out in a microplate format in an Envision plate reader. The FRET signal (emission of the acceptor fluorophore following excitation of the donor fluorophore) was detected over time following phagocytosis and expressed relative to the donor fluorophore fluorescence.

Phagosome acidification. BMDMs were prepared as above. Phagosomal $\mathrm{pH}$ was calculated by recording the ratio of the fluorescent emission at $520 \mathrm{~nm}$ of carboxyfluorescein succinimidyl ester (CFSE) conjugated to experimental particles excited at $490 \mathrm{~nm}$ and $450 \mathrm{~nm}$ followed by polynomial regression to a standard curve as previously detailed ${ }^{75,76}$.

Phagosome proteolysis. BMDMs were prepared as above. For assessment of the hydrolytic capacity of the phagosome, experimental particles were prepared bearing the proteolytic substrate, green DQ-BSA and the calibration fluorophore (Alexa Fluor $594 \mathrm{SE}$ ) as previously described 75,77 . The hydrolytic capacity of the cells was determined as the rate of substrate liberation of the DQ-Green-BSA from the experimental particles over time, relative to the calibration fluorophore. For comparison of hydrolytic capacities across experiments, the slopes (as described by the equation $y=\mathrm{mx}+\mathrm{c}$, where $y=\mathrm{RFU}, m=$ slope, and $x=$ time) of the linear portion of the relative substrate fluorescence plotted against time were calculated relative to the internal control indicated.

Phagosome ROS production. BMDMs were prepared as above. To determine the amount of $\mathrm{H}_{2} \mathrm{O}_{2}$ generated by macrophages stimulated with $50 \mu \mathrm{g} \mathrm{ml}^{-1}$ of serumopsonized zymosan (Sigma Aldrich), supernatant analysis was carried out by measuring the reaction of $20 \mu \mathrm{M}$ Amplex Red (Invitrogen \#A12222) in the presence of horse radish peroxidase using a FLUOstar Optima fluorescence plate reader (BMG Labtech) as previously described ${ }^{77,78}$. Hydrogen peroxide production is presented as relative to unstimulated macrophages.

Phagosome and lysosome isolation. WT and IFITM3 KO BMDMs were plated at $1 \times 10^{7}$ cells per dish in $70 \mathrm{~cm}^{2}$ Petri dishes and treated with $2400 \mathrm{IU} \mathrm{ml} \mathrm{m}^{-1} \mathrm{IFN} \beta$ for $24 \mathrm{~h}$ prior to and throughout the experiment. $7 \times 10^{7}$ cells $\left(7\right.$ dishes) and $4 \times 10^{7}$ cells (4 dishes) were used per condition for phagosome and lysosome isolation respectively. For phagosomes, magnetic latex beads $(2.79 \mu \mathrm{m}$, Bangs Laboratories \#UMC3001-UMC3N) were opsonized with IgG for $60 \mathrm{~min}$ at $37^{\circ} \mathrm{C}$. Beads were diluted in RPMI $+10 \%$ FBS and fed to BMDMs at a ratio of 10 beads per cell. Beads were allowed to phagocytose for $30 \mathrm{~min}$ before washing cells with PBS + and replacing with RPMI $+10 \%$ FBS for $4 \mathrm{~h}$. For lysosomes, $5 \mathrm{ml}$ media containing 2.5 $\mathrm{ml}$ iron dextran ( $\mathrm{FeDex}$, obtained as a gift from R. Yates) in $\mathrm{H}_{2} \mathrm{O}$ mixed with $2.5 \mathrm{ml}$ $2 \mathrm{X} \mathrm{RPMI}+10 \% \mathrm{FBS}$, was added to $5 \mathrm{ml}$ regular RPMI $+10 \% \mathrm{FBS}$, to make a total volume of $10 \mathrm{ml}$ used per dish. Cells were pulsed for $8 \mathrm{~h}$ with the FeDex solution before washing with PBS+ and chased by replacing with RPMI $+10 \%$ FBS for $1 \mathrm{~h}$.

Subsequent steps were performed on ice or in $4^{\circ} \mathrm{C}$. To isolate phagosomes and lysosomes, cells were washed twice with cold PBS+, replaced with $7 \mathrm{ml}$ cold PBS+, scraped, and collected in a $50 \mathrm{ml}$ tube. Cells were spun down at $450 \times g$ for $5 \mathrm{~min}$ 
and pellet was resuspended in $10 \mathrm{ml}$ homogenization buffer ( $250 \mathrm{mM}$ sucrose, 3 $\mathrm{mM}$ imidazole, $\mathrm{pH}$ 7.4) and spun down again. Final cell pellets were resuspended in $2.5 \mathrm{ml}$ homogenization buffer and homogenized using a $15 \mathrm{ml}$ Dounce homogenizer with a small clearance pestle for $20-30$ passes until $90 \%$ of cells were broken without nuclear breakage. Homogenate were spun down in $15 \mathrm{ml}$ tube at $135 \times g$ for $5 \mathrm{~min}$ and supernatant was collected in a $5 \mathrm{ml}$ propylene round bottom tube. To purify phagosomes and lysosomes, tubes with supernatant were placed in an EasySep magnet for $10 \mathrm{~min}$ before pouring out to discard unbound solution. Magnetic latex bead-containing phagosomes and FeDex-containing lysosomes attached to the side of the tube were washed and resuspended with $1 \mathrm{ml}$ homogenization buffer and spun down in a $1.5 \mathrm{ml}$ Eppendorf tube at $16,000 \times g$ for $15 \mathrm{~min}$. Pellets were resuspended with lysis buffer and stored at $-80^{\circ} \mathrm{C}$. Magnetic beads were removed by centrifugation prior to mass spectrometry.

Mass spectrometry. All sample preparation, LC-MS/MS data collection, and MS data searches were carried out at the SPARC BioCentre at The Hospital for Sick Children (Toronto, Canada). Samples were reduced with DTT $\left(10 \mathrm{mM}, 60^{\circ} \mathrm{C}, 1 \mathrm{~h}\right)$, alkylated with iodoacetamide (20 mM, room temperature, $45 \mathrm{~min}$, dark), and digested overnight at $37^{\circ} \mathrm{C}$ with trypsin $(2 \mu \mathrm{g}$; Pierce). Peptides were dried by vacuum centrifugation, desalted on C18 ziptips (Millipore) using a DigestPro MSi (Intavis Bioanalytical Instruments), and dried again by vacuum centrifugation before resuspension in Buffer A (0.1\% formic acid). Samples were analyzed by liquid chromatography-tandem mass spectrometry (LC-MS/MS) using an EASYnanoLC 1200 system with a $1 \mathrm{~h}$ analysis and an Orbitrap Fusion Lumos Tribrid Mass Spectrometer (Thermo Fisher Scientific). The LC portion of the analysis consisted of an 18 min linear gradient running 3-20\% of Buffer A to Buffer B $(0.1 \%$ FA, $80 \%$ acetonitrile), followed by a 31 min linear gradient running $20-35 \%$ of Buffer A to Buffer B, a 2 min ramp to $100 \%$ Buffer B and 9 min hold at 100\% Buffer $B$, all at a flow rate of $250 \mathrm{~nL} \mathrm{~min}^{-1}$. Samples were loaded into a $75 \mu \mathrm{m} \times 2 \mathrm{~cm}$ Acclaim PepMap 100 Pre-column followed by a $75 \mu \mathrm{m} \times 50 \mathrm{~cm}$ PepMax RSLC EASY-Spray analytical column filled with $2 \mu \mathrm{M} \mathrm{C18}$ beads (Thermo Fisher Scientific). MS1 acquisition resolution was set to 120,000 with an automatic gain control (AGC) target value of $4 \times 10^{5}$ and maximum ion injection time (IT) of 50 $\mathrm{ms}$ for a scan range of $\mathrm{m} / \mathrm{z} 375-1500$. Monoisotopic precursor selection (MIPS) was determined at the peptide level with a global intensity threshold of 10,000 and only peptides with charge states of $2-7$ were accepted, with dynamic exclusion set to $10 \mathrm{~s}$. Isolation for MS2 scans was performed in the quadrupole with an isolation window of $m / z 0.7$. MS2 scans were performed in the ion trap with maximum ion IT of $10 \mathrm{~ms}$, AGC target value of $1 \times 10^{4}$, and higher-energy collisional dissociation (HCD) activation with an NCE of 30. MS raw files were analyzed using Proteome Discoverer (version 2.2.0.388) and fragment lists and GO annotation searched against the mouse UniProt Reference database (downloaded Sept. 20, 2019; 54953 entries). For both search algorithms, the parent and fragment mass tolerances were set to $10 \mathrm{ppm}$ and $0.6 \mathrm{Da}$, respectively, and only complete tryptic peptides with a maximum of two missed cleavages were accepted. Protein abundance was compared between UT and IFN $\beta$-treated phagosomes and lysosomes. Complete proteomic datasets were deposited in the MassIVE repository, accession \# MSV000087014 [https://doi.org/10.25345/C5V51C].

Lysosome acidification. BMDMs were plated at $6 \times 10^{5}$ cells per well in 12 -well tissue culture plates with glass coverslips and treated with $2400 \mathrm{IU} \mathrm{ml}^{-1}$ IFN $\beta 24 \mathrm{~h}$ before treatment. Cells were pulsed with $10 \mathrm{kDa}$ FITC-dextran, washed and chased for $1 \mathrm{~h}$ in RPMI medium containing 10\% FBS and IFN $\beta$. Glass coverslips were transferred to an imaging chamber containing HEPES-buffered RPMI. The cells were visualized with a Leica DM IRB microscope. Once a cell was found that had bound fluorophore-labeled dextran, $\mathrm{pH}$ measurements were performed by fluorescence ratiometric imaging using a filter wheel (Sutter Instruments) to rapidly alternate between excitation filters. The FITC-dextran was excited by light from an EXFO X-cite 120 lamp (ExFo Life Sciences) transmitted through the appropriate excitation filters and directed to the sample using a dichroic mirror. The emitted light was captured by a CCD camera (Cascade II; Photometrics) after passing through an emission filter. MetaFluor (MDS Analytical Technologies) software was used to control the filter wheel and camera. An in situ calibration was performed for each lysosome after measurement. Samples were bathed in $\mathrm{K}^{+}$-rich buffers ranging from $\mathrm{pH} 4.5-9.6$ containing $10 \mu \mathrm{g} \mathrm{ml}^{-1}$ nigericin. Images were taken $5 \mathrm{~min}$ after the addition of each calibration solution to ensure equilibration to the desired $\mathrm{pH}$. The resulting fluorescence intensity ratio was plotted as a function of $\mathrm{pH}$ and fitted to a Boltzmann sigmoid that was used to interpolate ratios measured of lysosome acidification.

DQ-BSA degradation. BMDMs were plated at $1 \times 10^{5}$ cells per well in $\mu$-Slide 8 Well IBIDI chambers and treated with $2400 \mathrm{IU} \mathrm{ml}^{-1}$ IFN $\beta 24 \mathrm{~h}$ before infection. 4 $\mathrm{h}$ prior to infection, cells were pulsed with green DQ-BSA $\left(10 \mu \mathrm{g} \mathrm{ml}^{-1}\right)$ for $1 \mathrm{~h}$ and chased with RPMI medium containing $10 \%$ FBS and IFN $\beta$ for $3 \mathrm{~h}$. Cells were infected with 1:200 RFP-E. coli in RPMI. At $60 \mathrm{~min}$ p.i., all cells were washed three times with PBS+ and cultured in RPMI medium containing $10 \%$ FBS, IFN $\beta$ and $50 \mu \mathrm{g} \mathrm{ml}^{-1}$ gentamicin. $4 \mathrm{~h}$ p.i., cells were imaged live using a spinning disk confocal microscope equipped with a $\times 63$ objective. DQ-BSA-positive bacteria were quantified using Volocity 6 software.
actA:GFP Lm protein expression. J774 murine macrophage-like cells were plated at $2 \times 10^{6}$ cells per well in 6 -well tissue culture plates overnight in DMEM. Cells were infected with mid-log bacterial cultures resuspended 1:20 in 100 $\mu$ DMEM for $10 \mathrm{~min}$. Infected cells were washed with PBS+ after $60 \mathrm{~min}$ and replaced with DMEM containing $50 \mu \mathrm{g} \mathrm{ml}^{-1}$ gentamicin. At each time point, cells were lysed with $0.1 \%$ Triton $\mathrm{X}-100$ in PBS+, bacterial titers in the lysates were determined by plating dilutions for $\mathrm{CFU}$ of bacteria recovered via centrifugation. Bacteria were normalized to $\mathrm{CFU}$ and whole-cell lysates were generated by $3 \mathrm{mg} \mathrm{ml}^{-1}$ lysozyme treatment in Tris-EDTA for $60 \mathrm{~min}$ at $37^{\circ} \mathrm{C}$ and subjected to western blot analysis.

ActA, InIB, and LLO protein expression. WT and IFITM3 KO BMDMs were plated at $2.5 \times 10^{6}$ cells per well in 6 -well tissue culture plates and treated with $2400 \mathrm{IU} \mathrm{ml}^{-1}$ IFN $\beta 24 \mathrm{~h}$ before infection. Cells were infected with WT, TKO or $\triangle a c t A p l c A p l c B$ Lm at an MOI of 10 in RPMI. At $60 \mathrm{~min}$ p.i., all cells were washed three times with PBS+ and cultured in RPMI medium containing 10\% FBS, $50 \mu \mathrm{g}$ $\mathrm{ml}^{-1}$ gentamicin IFN $\beta$. Cells were lysed with $1 \%$ Triton X-100 in $1 \mathrm{ml} \mathrm{PBS}+$ at 1 , 2, and $4 \mathrm{~h}$ p.i. $100 \mu \mathrm{l}$ of lysate was used for serial dilution and plated on BHI agar plate to obtain bacterial CFU. Remaining lysates were spun down to recover soluble fraction for LLO and bacteria pellet for ActA and InlB. Samples were diluted and resuspended in sample buffer, boiled for $5 \mathrm{~min}$, and subjected to western blot analysis.

ActA degradation. RAW264.7 macrophages, WT and IFITM3 KO BMDMs were plated at $3 \times 10^{5}$ cells per well in 24 -well tissue culture plates. Where indicated, cells were treated with $2400 \mathrm{IU} \mathrm{ml}^{-1} \mathrm{IFN} \beta, 100 \mathrm{nM}$ bafilomycin A, or protease inhibitors $24 \mathrm{~h}, 4 \mathrm{~h}$ and $30 \mathrm{~min}$ respectively before infection. Cells were infected with actA:GFP reporter strains; WT, $\Delta h l y \mathrm{Lm}$ and TKO Lm at an MOI of 10 in RPMI At 60 min p.i., all cells were washed three times with PBS+ and cultured in RPMI medium containing $10 \%$ FBS, IFN $\beta$ and $50 \mu \mathrm{g} \mathrm{ml}^{-1}$ gentamicin. At $4 \mathrm{~h}$ p.i., cells were fixed with $2.5 \%$ PFA for $10 \mathrm{~min}$. To visualize bacteria surface protein degradation in phagosomes, cells were stained with antibodies to ActA and LAMP1. Cells were imaged using a spinning disk confocal microscope equipped with a $\times 63$ objective. ActA- and LAMP1-positive actA:GFP reporter-positive bacteria were quantified using Volocity 6 software.

Actin recruitment. WT and IFITM3 KO BMDMs were plated at $3 \times 10^{5}$ cells per well in 24-well tissue culture plates and treated with $2400 \mathrm{IU} \mathrm{ml}^{-1}$ IFN $\beta 24 \mathrm{~h}$ before infection. Cells were infected with WT $L m$ at an MOI of 20 in RPMI. At 60 min p.i., all cells were washed three times with PBS+ and cultured in RPMI medium containing $10 \% \mathrm{FBS}, 50 \mu \mathrm{g} \mathrm{ml}^{-1}$ gentamicin IFN $\beta$. At $1,1.25,1.5,1.75,2$ and $4 \mathrm{~h}$ p.i., cells were fixed with $2.5 \%$ PFA. Differential antibody staining was carried out to stain extracellular bacteria before permeabilizing the cells to stain for total bacteria. BMDMs were also stained for actin using phalloidin. Cells were imaged on a Quorum spinning disk confocal scan head equipped with a $\times 63$ objective. Actin recruitment was quantified as phalloidin-positive internalized bacteria (total bacteria minus extracellular bacteria) over the time course, and motile $\mathrm{Lm}$ (actin comet tail-positive bacteria) were quantified $4 \mathrm{~h}$ p.i., using Volocity 6 software.

Infection focus assay. Assay was conducted as previously described ${ }^{57}$. Briefly, WT and IFITM3 KO BMDMs were plated at $1.5 \times 10^{6}$ cells per well in 24-well tissue culture plates and treated with $2400 \mathrm{IU} \mathrm{ml}^{-1}$ IFN $\beta 24 \mathrm{~h}$ before infection. AML12, MEF and Caco- 2 cells were plated at 2, 4, and $2.5 \times 10^{5}$ cells per well, respectively, in 24-well tissue culture plates and treated with $2400 \mathrm{IU} \mathrm{ml}^{-1}$ IFN $\beta 24 \mathrm{~h}$ before infection. Cells were infected with WT $L m$ at an MOI of 0.001 (BMDM), 1 (AML12), 0.5 (MEFs) or 0.1 (Caco-2) in RPMI (BMDM) or DMEM (all others). At $18 \mathrm{~h}$ p.i., cells were fixed and counterstained with $\mathrm{Lm}$ and DAPI, and imaged on a Quorum spinning disk confocal scan head equipped with a $\times 10$ objective. The number of infected cells per focus of infection was quantified using Volocity 6 software.

Replication assay. WT and IFITM3 KO BMDMs were plated at $3 \times 10^{5}$ cells per well (sub-confluence density) in 24-well tissue culture plates and treated with 2400 $\mathrm{IU} \mathrm{ml}^{-1}$ IFN $\beta 24 \mathrm{~h}$ before infection. Cells were infected with WT or $\triangle a c t A \mathrm{Lm}$ at an MOI of 1 in RPMI. At 60 min p.i., all cells were washed three times with PBS+ and cultured in RPMI medium containing $10 \% \mathrm{FBS}, 50 \mu \mathrm{g} \mathrm{ml}{ }^{-1}$ gentamicin IFN $\beta$. At 2 , 4,8 and $12 \mathrm{~h}$ p.i., cells were lysed with PBS+ containing $1 \%$ Triton X-100. Serial dilutions of the lysates were plated on $\mathrm{BHI}$ agar plates and incubated at $37^{\circ} \mathrm{C}$ for $16 \mathrm{~h}$ for subsequent quantification of bacterial CFU.

In vivo infection. 8-12-week-old sex- and age-matched WT, IFITM3 KO, and IFNAR1 KO mice were used for WT $L m$ infection. Mice were restrained using Mouse Tail Illuminator Restrainer (Braintree Scientific), tail was swabbed with 70\% $\mathrm{EtOH}$ and warmed to dilate lateral tail vein. Mice were infected with $5 \times 10^{4}$ bacteria diluted in $200 \mu \mathrm{l}$ of PBS + via intravenous injection using $30 \mathrm{G} \mathrm{x} 1 / 2$ needles (BD). Mice were monitored and weighed 3 times a day at $9 \mathrm{am}, 3 \mathrm{pm}$ and $9 \mathrm{pm}$. Survival rate was plotted using Kaplen-Meier function after 10 days p.i. Organs used to determine bacterial load were harvested from a separate group of mice $72 \mathrm{~h}$ 
p.i. Mice were euthanized by $\mathrm{CO}_{2}$, and liver, spleen and brain were obtained and homogenized in $1 \mathrm{ml} \mathrm{PBS}+$, and serial dilutions of the tissue were plated on $\mathrm{BHI}$ agar plates and incubated at $37^{\circ} \mathrm{C}$ for $16 \mathrm{~h}$ for subsequent quantification of CFU. Final bacterial load was calculated after normalization to tissue weight.

Tissue staining. Liver obtained from WT and IFITM3 KO mice infected with WT $L m$ or GFP- $L m$ for $72 \mathrm{~h}$ was embedded in OCT medium (Tissue-Tek \#4583) and snap-frozen in liquid nitrogen. $5 \mu \mathrm{m}$ sections collected in 4 series were obtained using a Cryostat (Leica CM 1850) and mounted on charged slides (VWR \#CA48311-703). Tissue was fixed in ice-cold methanol for $10 \mathrm{~min}$ at $-20^{\circ} \mathrm{C}$. For hematoxylin and eosin staining, slides were rehydrated and stained according to the following: Hematoxylin (3 min), $\mathrm{H}_{2} \mathrm{O}(1 \mathrm{~min})$, Clarifier (1 min), $\mathrm{H}_{2} \mathrm{O}$ (1 min), Bluing Reagent $(3 \mathrm{~min}), \mathrm{H}_{2} \mathrm{O}(1 \mathrm{~min}), 95 \% \mathrm{EtOH}$ (rinse), Eosin $(30 \mathrm{~s}), 75 \% \mathrm{EtOH}$ (rinse), $95 \% \mathrm{EtOH}$ (rinse), $100 \% \mathrm{EtOH}(1 \mathrm{~min} \times 3)$, Xylene $(1 \mathrm{~min} \times 3)$. Tissue was sealed with mounting media (Sigma \#107960) and liver infection foci were imaged using Leica DM1000LED with a $\times 20$ objective. Number of foci were calculated per square $\mathrm{mm}$ of tissue. For immunofluorescence staining, slides were blocked with $5 \%$ BSA, $10 \%$ goat serum and $0.1 \%$ Triton X-100 diluted in PBS+ for $30 \mathrm{~min}$ at RT. Tissue were stained with antibody to $\mathrm{Lm}$, ActA, CD3e and $\mathrm{F} 4 / 80$ overnight at $4{ }^{\circ} \mathrm{C}$, washed with PBS + and counterstained with secondary antibody and DAPI. Tissue was imaged on a Quorum spinning disk confocal scan head equipped with a $\times 10$ and $\times 60$ objective. ActA and immune cell recruitment, and the number of infected cells per focus of infection were quantified using Volocity 6 software.

IFN $\beta$ production. WT and IFITM3 KO BMDMs were plated at $4 \times 10^{6}$ cells per well in 6-well tissue culture plates and treated with $2400 \mathrm{IU} \mathrm{ml}^{-1}$ IFN $\beta 24 \mathrm{~h}$ before infection. Cells were infected with WT Lm at an MOI of 0.001 in RPMI. At 60 min p.i., all cells were washed three times with PBS+ and cultured in RPMI medium containing $10 \%$ FBS and $50 \mu \mathrm{g} \mathrm{ml}^{-1}$ gentamicin. Cell culture supernatant was collected 4, 8, 12 and $24 \mathrm{~h}$ p.i. IFN $\beta$ levels were measured using ELISA kit (PBL Assay Science \#4200-1).

RNA isolation and qPCR. Liver tissue from WT and IFITM3 KO mice infected with WT $L m$ was obtained and RNA was isolated using the RNeasy kit (QIAGEN \#74104). cDNA was synthesized using iScript Reverse Transcription Supermix for reverse transcription. $10 \mathrm{ng}$ of cDNA per reaction was used for quantitative PCR using SsoFast EvaGreen Supermix (Bio-Rad \#1708840) with Hprt as the housekeeping gene. The following primers were obtained from Sigma: Ifnb (FCCCTATGGAGATGACGGAGA R-CTGTCTGCTGGTGGAGTTCA), Ifngrl (FGTAGCCTCACCGCCTATCAC R-GGGCCTCTCCTGTGAGTCTA), Tnfa (FCCCATGGATGTCCCATTTAG R-CAATCAGGAGGGTGTGTGTG), Il4 (FGCCTGCTTTTCACATGAGGT R-AAATATGCGAAGCACCTTGG), Il10 (FCCAAGCCTTATCGGAAATGA R-TTTTCACAGGGGAGAAATCG), $I l 12$ (FAGCATAAGAGACGCCCTCAA R-GGTGTTACAGGCCCAAAGAA). All primers used are listed in Supplementary Table 1.

Statistical analysis. Statistical analyses and graph plotting were conducted using GraphPad Prism v.8.4.1. The mean \pm s.d. is shown in figures, and $P$ values were calculated as described in figure legends. $P<0.05$ was considered statistically significant. Unless stated otherwise, ${ }^{*} P<0.05$, ${ }^{* *} P<0.01$, $* * * P<0.001$, **** $P<$ 0.0001, not significant (ns) $P>0.05$.

Reporting summary. Further information on research design is available in the Nature Research Reporting Summary linked to this article.

\section{Data availability}

The data that support the findings of this study are available from the corresponding authors upon request. Complete proteomic datasets were deposited in the MassIVE repository, accession \# MSV000087014 [https://doi.org/10.25345/C5V51C]. Source data are provided with this paper.

Received: 22 April 2021; Accepted: 18 July 2021; Published online: 17 August 2021

\section{References}

1. Smith, G. A. et al. The two distinct phospholipases C of Listeria monocytogenes have overlapping roles in escape from a vacuole and cell-to-cell spread. Infect. Immun. 63, 4231-4237 (1995)

2. Bielecki, J., Youngman, P., Connelly, P. \& Portnoy, D. A. Bacillus subtilis expressing a haemolysin gene from Listeria monocytogenes can grow in mammalian cells. Nature 345, 175-176 (1990).

3. Boujemaa-Paterski, R. et al. Listeria protein ActA mimics WASp family proteins: it activates filament barbed end branching by Arp $2 / 3$ complex. Biochemistry 40, 11390-11404 (2001)
4. Gutierrez, M. G. et al. Autophagy is a defense mechanism inhibiting BCG and Mycobacterium tuberculosis survival in infected macrophages. Cell 119 , 753-766 (2004)

5. Birmingham, C. L. et al. Listeria monocytogenes evades killing by autophagy during colonization of host cells. Autophagy 3, 442-451 (2007).

6. Perrin, A. J., Jiang, X., Birmingham, C. L., So, N. S. \& Brumell, J. H. Recognition of bacteria in the cytosol of Mammalian cells by the ubiquitin system. Curr. Biol. 14, 806-811 (2004).

7. Czuczman, M. A. et al. Listeria monocytogenes exploits efferocytosis to promote cell-to-cell spread. Nature 509, 230-234 (2014).

8. Pestka, S., Krause, C. D. \& Walter, M. R. Interferons, interferon-like cytokines, and their receptors. Immunol. Rev. 202, 8-32 (2004).

9. Gonzalez-Navajas, J. M., Lee, J., David, M. \& Raz, E. Immunomodulatory functions of type I interferons. Nat. Rev. Immunol. 12, 125-135 (2012).

10. Mesev, E. V., LeDesma, R. A. \& Ploss, A. Decoding type I and III interferon signalling during viral infection. Nat. Microbiol 4, 914-924 (2019).

11. Woodward, J. J., Iavarone, A. T. \& Portnoy, D. A. c-di-AMP secreted by intracellular Listeria monocytogenes activates a host type I interferon response. Science 328, 1703-1705 (2010).

12. Abdullah, Z. et al. RIG-I detects infection with live Listeria by sensing secreted bacterial nucleic acids. EMBO J. 31, 4153-4164 (2012).

13. McFarland, A. P. et al. Sensing of bacterial cyclic dinucleotides by the oxidoreductase RECON promotes NF-kappaB activation and shapes a proinflammatory antibacterial state. Immunity 46, 433-445 (2017).

14. O'Connell, R. M. et al. Type I interferon production enhances susceptibility to Listeria monocytogenes infection. J. Exp. Med. 200, 437-445 (2004).

15. Auerbuch, V., Brockstedt, D. G., Meyer-Morse, N., O’Riordan, M. \& Portnoy, D. A. Mice lacking the type I interferon receptor are resistant to Listeria monocytogenes. J. Exp. Med. 200, 527-533 (2004).

16. Shaabani, N. et al. IFN- $\beta$, but not IFN- $\alpha$, is responsible for the pro-bacterial effect of type I interferon. Cell Physiol. Biochem. 55, 256-264 (2021).

17. McNab, F., Mayer-Barber, K., Sher, A., Wack, A. \& O'Garra, A. Type I interferons in infectious disease. Nat. Rev. Immunol. 15, 87-103 (2015).

18. Brzoza-Lewis, K. L., Hoth, J. J. \& Hiltbold, E. M. Type I interferon signaling regulates the composition of inflammatory infiltrates upon infection with Listeria monocytogenes. Cell Immunol. 273, 41-51 (2012).

19. Jia, T., Leiner, I., Dorothee, G., Brandl, K. \& Pamer, E. G. MyD88 and Type I interferon receptor-mediated chemokine induction and monocyte recruitment during Listeria monocytogenes infection. J. Immunol. 183, 1271-1278 (2009).

20. Osborne, S. E. et al. Type I interferon promotes cell-to-cell spread of Listeria monocytogenes. Cell Microbiol 19, e12660 (2017).

21. Schoggins, J. W. et al. A diverse range of gene products are effectors of the type I interferon antiviral response. Nature 472, 481-485 (2011).

22. Portnoy, D. A., Jacks, P. S. \& Hinrichs, D. J. Role of hemolysin for the intracellular growth of Listeria monocytogenes. J. Exp. Med. 167, 1459-1471 (1988).

23. Flannagan, R. S., Cosio, G. \& Grinstein, S. Antimicrobial mechanisms of phagocytes and bacterial evasion strategies. Nat. Rev. Microbiol. 7, 355-366 (2009).

24. Ewanchuk, B. W. \& Yates, R. M. The phagosome and redox control of antigen processing. Free Radic. Biol. Med. 125, 53-61 (2018).

25. Yates, R. M., Hermetter, A., Taylor, G. A. \& Russell, D. G. Macrophage activation downregulates the degradative capacity of the phagosome. Traffic $\mathbf{8}$, 241-250 (2007).

26. Trost, $M$. et al. The phagosomal proteome in interferon-gamma-activated macrophages. Immunity 30, 143-154 (2009).

27. Rybicka, J. M., Balce, D. R., Khan, M. F., Krohn, R. M. \& Yates, R. M. NADPH oxidase activity controls phagosomal proteolysis in macrophages through modulation of the lumenal redox environment of phagosomes. Proc. Natl Acad. Sci. USA 107, 10496-10501 (2010).

28. Savina, A. et al. NOX2 controls phagosomal $\mathrm{pH}$ to regulate antigen processing during crosspresentation by dendritic cells. Cell 126, 205-218 (2006).

29. Huang, S. et al. Immune response in mice that lack the interferon-gamma receptor. Science 259, 1742-1745 (1993)

30. Portnoy, D. A., Schreiber, R. D., Connelly, P. \& Tilney, L. G. Gamma interferon limits access of Listeria monocytogenes to the macrophage cytoplasm. J. Exp. Med. 170, 2141-2146 (1989).

31. Lam, G. Y., Huang, J. \& Brumell, J. H. The many roles of NOX2 NADPH oxidase-derived ROS in immunity. Semin Immunopathol. 32, 415-430 (2010)

32. Cheung, S., Greene, C. \& Yates, R. M. Simultaneous analysis of multiple lumenal parameters of individual phagosomes using high-content imaging. Methods Mol. Biol. 1519, 227-239 (2017)

33. Arnett, E. et al. The pore-forming toxin listeriolysin $\mathrm{O}$ is degraded by neutrophil metalloproteinase- 8 and fails to mediate Listeria monocytogenes intracellular survival in neutrophils. J. Immunol. 192, 234-244 (2014).

34. Gouin, E., Welch, M. D. \& Cossart, P. Actin-based motility of intracellular pathogens. Curr. Opin. Microbiol. 8, 35-45 (2005)

35. Poussin, M. A. \& Goldfine, H. Evidence for the involvement of ActA in maturation of the Listeria monocytogenes phagosome. Cell Res. 20, 109-112 (2010). 
36. Wong, K. K., Bouwer, H. G. \& Freitag, N. E. Evidence implicating the 5 untranslated region of Listeria monocytogenes actA in the regulation of bacterial actin-based motility. Cell Microbiol. 6, 155-166 (2004).

37. Moors, M. A., Levitt, B., Youngman, P. \& Portnoy, D. A. Expression of listeriolysin $\mathrm{O}$ and ActA by intracellular and extracellular Listeria monocytogenes. Infect. Immun. 67, 131-139 (1999).

38. Freitag, N. E. \& Jacobs, K. E. Examination of Listeria monocytogenes intracellular gene expression by using the green fluorescent protein of Aequorea victoria. Infect. Immun. 67, 1844-1852 (1999).

39. Lukacs, G. L., Rotstein, O. D. \& Grinstein, S. Phagosomal acidification is mediated by a vacuolar-type $\mathrm{H}(+)$-ATPase in murine macrophages. J. Biol. Chem. 265, 21099-21107 (1990).

40. Shen, Y., Naujokas, M., Park, M. \& Ireton, K. InIB-dependent internalization of Listeria is mediated by the Met receptor tyrosine kinase. Cell 103, 501-510 (2000).

41. Siegrist, M. S. et al. Host actin polymerization tunes the cell division cycle of an intracellular pathogen. Cell Rep. 11, 499-507 (2015).

42. Glowacka, I. et al. Evidence that TMPRSS2 activates the severe acute respiratory syndrome coronavirus spike protein for membrane fusion and reduces viral control by the humoral immune response. J. Virol. $\mathbf{8 5}$, 4122-4134 (2011).

43. Ou, X. et al. Characterization of spike glycoprotein of SARS-CoV-2 on virus entry and its immune cross-reactivity with SARS-CoV. Nat. Commun. 11, 1620 (2020).

44. Hoffmann, M. et al. SARS-CoV-2 cell entry depends on ACE2 and TMPRSS2 and is blocked by a clinically proven protease inhibitor. Cell 181, 271-280 (2020).

45. Chandran, K., Sullivan, N. J., Felbor, U., Whelan, S. P. \& Cunningham, J. M. Endosomal proteolysis of the Ebola virus glycoprotein is necessary for infection. Science 308, 1643-1645 (2005).

46. Diaz-Salinas, M. A., Silva-Ayala, D., Lopez, S. \& Arias, C. F. Rotaviruses reach late endosomes and require the cation-dependent mannose-6-phosphate receptor and the activity of cathepsin proteases to enter the cell. J. Virol. 88, 4389-4402 (2014).

47. Edinger, T. O., Pohl, M. O., Yanguez, E. \& Stertz, S. Cathepsin W is required for escape of influenza A virus from late endosomes. mBio 6, e00297 (2015).

48. Brass, A. L. et al. The IFITM proteins mediate cellular resistance to influenza A H1N1 virus, West Nile virus, and dengue virus. Cell 139, 1243-1254 (2009).

49. Everitt, A. R. et al. IFITM3 restricts the morbidity and mortality associated with influenza. Nature 484, 519-523 (2012).

50. Lee, J. et al. IFITM3 functions as a PIP3 scaffold to amplify PI3K signalling in B cells. Nature 588, 491-497 (2020).

51. Feeley, E. M. et al. IFITM3 inhibits influenza A virus infection by preventing cytosolic entry. PLoS Pathog. 7, e1002337 (2011).

52. $\mathrm{Yu}$, J. et al. IFITM proteins restrict HIV-1 infection by antagonizing the envelope glycoprotein. Cell Rep. 13, 145-156 (2015).

53. Anafu, A. A., Bowen, C. H., Chin, C. R., Brass, A. L. \& Holm, G. H. Interferoninducible transmembrane protein 3 (IFITM3) restricts reovirus cell entry. J. Biol. Chem. 288, 17261-17271 (2013).

54. Naujoks, J. et al. IFNs modify the proteome of Legionella-containing vacuoles and restrict infection via IRG1-derived itaconic acid. PLoS Pathog. 12, e1005408 (2016).

55. Poddar, S., Hyde, J. L., Gorman, M. J., Farzan, M. \& Diamond, M. S. The interferon-stimulated gene IFITM3 restricts infection and pathogenesis of arthritogenic and encephalitic alphaviruses. J. Virol. 90, 8780-8794 (2016).

56. Hur, J. et al. The innate immunity protein IFITM3 modulates $\gamma$-secretase in Alzheimer's disease. Nature 586, 735-740 (2020).

57. Tan, J. M. J. et al. An ATG16L1-dependent pathway promotes plasma membrane repair and limits Listeria monocytogenes cell-to-cell spread. Nat. Microbiol. 3, 1472-1485 (2018).

58. Zwaferink, H., Stockinger, S., Hazemi, P., Lemmens-Gruber, R. \& Decker, T. IFN-beta increases listeriolysin O-induced membrane permeabilization and death of macrophages. J. Immunol. 180, 4116-4123 (2008).

59. Carrero, J. A., Calderon, B. \& Unanue, E. R. Type I interferon sensitizes lymphocytes to apoptosis and reduces resistance to Listeria infection. J. Exp. Med. 200, 535-540 (2004).

60. Carrero, J. A., Calderon, B. \& Unanue, E. R. Listeriolysin O from Listeria monocytogenes is a lymphocyte apoptogenic molecule. J. Immunol. 172, 4866-4874 (2004).

61. Alteber, Z. et al. The anti-inflammatory IFITM genes ameliorate colitis and partially protect from tumorigenesis by changing immunity and microbiota. Immunol. Cell Biol. 96, 284-297 (2018).

62. Shen, C. et al. A functional promoter polymorphism of IFITM3 is associated with susceptibility to pediatric tuberculosis in Han Chinese population. PLoS One 8, e67816 (2013).
63. Liu, X. et al. IFITM3 promotes bone metastasis of prostate cancer cells by mediating activation of the TGF-beta signaling pathway. Cell Death Dis. 10, 517 (2019).

64. Boxx, G. M. \& Cheng, G. The roles of type I interferon in bacterial infection. Cell Host Microbe. 19, 760-769 (2016).

65. Nakamura, S., Davis, K. M. \& Weiser, J. N. Synergistic stimulation of type I interferons during influenza virus coinfection promotes Streptococcus pneumoniae colonization in mice. J. Clin. Invest. 121, 3657-3665 (2011).

66. Kudva, A. et al. Influenza A inhibits Th17-mediated host defense against bacterial pneumonia in mice. J. Immunol. 186, 1666-1674 (2011).

67. Zhang, H., Zoued, A., Liu, X., Sit, B. \& Waldor, M. K. Type I interferon remodels lysosome function and modifies intestinal epithelial defense. Proc. Natl Acad. Sci. USA 117, 29862-29871 (2020).

68. Everitt, A. R. et al. Defining the range of pathogens susceptible to Ifitm 3 restriction using a knockout mouse model. PLoS One 8, e80723 (2013).

69. Horton, R. M., Hunt, H. D., Ho, S. N., Pullen, J. K. \& Pease, L. R. Engineering hybrid genes without the use of restriction enzymes: gene splicing by overlap extension. Gene 77, 61-68 (1989).

70. Shen, A. \& Higgins, D. E. The 5' untranslated region-mediated enhancement of intracellular listeriolysin O production is required for Listeria monocytogenes pathogenicity. Mol. Microbiol. 57, 1460-1473 (2005).

71. Gorman, M. J., Poddar, S., Farzan, M. \& Diamond, M. S. The interferonstimulated gene Ifitm 3 restricts west nile virus infection and pathogenesis. J. Virol. 90, 8212-8225 (2016).

72. Reniere, M. L. et al. Glutathione activates virulence gene expression of an intracellular pathogen. Nature 517, 170-173 (2015).

73. Ostrowski, P. P., Fairn, G. D., Grinstein, S. \& Johnson, D. E. Cresyl violet: a superior fluorescent lysosomal marker. Traffic 17, 1313-1321 (2016).

74. Balce, D. R., Allan, E. R., McKenna, N. \& Yates, R. M. gamma-Interferoninducible lysosomal thiol reductase (GILT) maintains phagosomal proteolysis in alternatively activated macrophages. J. Biol. Chem. 289, 31891-31904 (2014).

75. Yates, R. M. \& Russell, D. G. Real-time spectrofluorometric assays for the lumenal environment of the maturing phagosome. Methods Mol. Biol. 445, 311-325 (2008).

76. Yates, R. M., Hermetter, A. \& Russell, D. G. The kinetics of phagosome maturation as a function of phagosome/lysosome fusion and acquisition of hydrolytic activity. Traffic 6, 413-420 (2005).

77. Balce, D. R. \& Yates, R. M. Redox-sensitive probes for the measurement of redox chemistries within phagosomes of macrophages and dendritic cells. Redox Biol. 1, 467-474 (2013).

78. Yates, R. M. Redox considerations in the phagosome: current concepts, controversies, and future challenges. Antioxid. Redox Signal 18, 628-629 (2013).

\section{Acknowledgements}

We are grateful to P. Cossart, N. Freitag, P. Lauer, M. Loessner, and D. Portnoy for providing reagents, to P. Paroutis for help with confocal microscopy, and to the SickKids SPARC BioCentre for help with mass spectrometry. J.H.B. holds the Pitblado Chair in Cell Biology. Infrastructure for the Brumell Laboratory was provided by a John Evans Leadership Fund grant from the Canadian Foundation for Innovation and the Ontario Innovation Trust. D.A.A. was supported by a studentship from the Research Training Committee at the Hospital for Sick Children and an NSERC CGS-D scholarship. M.C. was supported by NSERC PGS-D scholarship and CIHR Training Fellowship (TGF53877). This work was supported by operating grants from the Canadian Institutes of Health Research (FDN\#154329 to J.H.B, FDN\#143202 to S.G. and MOP\#119289 to B.R) and the National Institutes of Health (\#R01AI146102 to D.E.H. and \#R01AI145296 to M. S.D.). B.R. holds a Canada Research Chair in Proteomics and Molecular Medicine.

\section{Author contributions}

J.H.B., D.E.H., J.M.J.T., and J.M.R., designed the experiments. J.M.J.T., M.E.G., J.M.R., C.J.G., J.D.R.M., D.A.A., A.R.R.M., T.L., K.J.W., M.C. and P.P.O performed the experiments. B.R., M.S.D., S.G., and R.M.Y. contributed reagents and experimental advice.

\section{Competing interests}

M.S.D. is a consultant for Inbios, Vir Biotechnology, NGM Biopharmaceuticals, and Carnival Corporation, and on the Scientific Advisory Boards of Moderna and Immunome. The Diamond laboratory has received funding support in sponsored research agreements from Moderna, Vir Biotechnology, and Emergent BioSolutions. The remaining authors declare no competing interests. 


\section{Additional information}

Supplementary information The online version contains supplementary material available at https://doi.org/10.1038/s41467-021-24982-0.

Correspondence and requests for materials should be addressed to D.E.H. or J.H.B.

Peer review information Nature Communications thanks Matthias Trost and the other, anonymous, reviewer(s) for their contribution to the peer review of this work.

Reprints and permission information is available at http://www.nature.com/reprints

Publisher's note Springer Nature remains neutral with regard to jurisdictional claims in published maps and institutional affiliations. (c) (1) Open Access This article is licensed under a Creative Commons Attribution 4.0 International License, which permits use, sharing, adaptation, distribution and reproduction in any medium or format, as long as you give appropriate credit to the original author(s) and the source, provide a link to the Creative Commons license, and indicate if changes were made. The images or other third party material in this article are included in the article's Creative Commons license, unless indicated otherwise in a credit line to the material. If material is not included in the article's Creative Commons license and your intended use is not permitted by statutory regulation or exceeds the permitted use, you will need to obtain permission directly from the copyright holder. To view a copy of this license, visit http://creativecommons.org/ licenses/by/4.0/.

(C) The Author(s) 2021 